CALT-68-2258

CITUSC/00-005

PUPT-1904

UMDEPP 00-046

\title{
Two Two-Dimensional Supergravity Theories from Calabi-Yau Four-Folds
}

\author{
S. James Gates, Jr., \\ - Department of Physics, University of Maryland at College Park, \\ College Park, MD 20742-4111, USA \\ * California Institute of Technology, Pasadena, CA 91125, USA, \\ CIT-USC Center For Theoretical Physics \\ $\diamond$ Joseph Henry Laboratories, Princeton University, \\ Princeton, NJ 08544, USA \\ $\odot$ School of Natural Sciences, Institute for Advanced Study, \\ Olden Lane, Princeton, NJ 08540, USA
}

We consider two-dimensional supergravity theories with four supercharges constructed from compactification of Type II string theory on a generic Calabi-Yau four-fold. In Type IIA and Type IIB cases, respectively, new superspace formulations of $\mathcal{N}=(2,2)$ and $\mathcal{N}=(0,4)$ dilaton supergravities are found and their coupling to matter multiplets is discussed.

May 2000

\footnotetext{
1 E-Mail: gatess@wam.umd.edu

2 E-Mail: gukov@feynman.princeton.edu
} 


\section{Introduction}

For a long time, compactification of heterotic string theory on Calabi-Yau manifolds was the primary candidate for constructing realistic models in four dimensions with $\mathcal{N}=1$ supersymmetry. This was also a strong motivation to study Type II superstrings on CalabiYau three-folds which share many common properties with the corresponding heterotic compactifications. At the same time substantial progress has been made in understanding the mathematical aspects of Calabi-Yau three-folds, such as quantum cohomology and mirror symmetry [1]. It was not until the discovery of F-theory [2] that it was realized that $\mathcal{N}=1$ four-dimensional heterotic string vacua can be equivalently described as F-theory compactifications on elliptically fibered Calabi-Yau four-folds. Since then, the study of Calabi-Yau four-fold compactifications has become of particular importance for physical applications. Compactification of F-theory on an elliptically fibered Calabi-Yau four-fold is closely related to the corresponding compactifications of Type IIA and M-theory. Namely, when the area of the elliptic fiber shrinks to zero, M-theory compactification on a CalabiYau four-fold is well described by F-theory compactification on the same Calabi-Yau manifold. On the other hand, Type IIA string theory is related to M-theory via compactification on an extra circle (of small radius).

One of the most striking outcomes in the study of compactifications on Calabi-Yau three-folds is the great success in understanding non-perturbative phenomena in $\mathcal{N}=2$ field theories in four dimensions, see e.g. [3] for introduction and references. On the other hand, understanding of Calabi-Yau four-fold compactifications still is quite far from that stage, so we shall not discuss non-perturbative phenomena in this paper. Instead, we consider classical supergravity theories interacting with two-dimensional non-linear sigmamodels that can be constructed from Calabi-Yau four-folds. Surprisingly, it turns out that manifestly supersymmetric formulations of such theories has not been given previously.

On general grounds, a compactification of Type IIA (IIB) string theory on a CalabiYau four-fold leads to a $\mathcal{N}=(2,2)$ (resp. $\mathcal{N}=(0,4))$ effective field theory in two dimensions. In the low-energy limit the theory is described by supergravity coupled to matter. For example, from the Kaluza-Klein reduction of Type IIA string theory on a CalabiYau four-fold in section 3 we find that a suitable low-energy theory is $\mathcal{N}=(2,2)$ dilaton

supergravity interacting with some number of chiral and twisted chiral multiplets. It is invariant under the "mirror transformation" which, acting on the matter fields, exchanges chiral multiplets and twisted chiral multiplets. We thus generalize the proposal of [4] where 
it was suggested that the "kinematic structure" of the mirror transformation has its origin in a mapping between chiral and twisted chiral multiplets when these superfields are regarded as the fundamental degrees of $\mathcal{N}=(2,2)$ superstring theories. The generalization posits that this mapping also applies to the effective action. Another characteristic feature of this supergravity theory is that the supergravity multiplet contains a real dilaton field. In a special case, when all matter multiplets are chiral and massless, a component action of this $\mathcal{N}=2$ dilaton supergravity was constructed in [5]. However, to describe Type IIA compactifications on Calabi-Yau four-folds we need a generalization of this theory that includes interaction with twisted chiral multiplets and the possibility to turn on the superpotential and as well the twisted superpotential. Thus, in sections 4 and 5 we present superspace construction of general $\mathcal{N}=(2,2)$ dilaton supergravity coupled to matter. The construction in section 4 is based on the Goldstone mechanism in the superspace formulation of non-minimal gauged $\mathcal{N}=(2,2)$ supergravity. Coupling of the new $\mathcal{N}=(2,2)$ dilaton supergravity to matter multiplets is the subject of section 5 , where we discuss local integration in superspace. In section 6 we perform the Kaluza-Klein reduction of Type IIB string theory on a Calabi-Yau four-fold and describe the component action of the resulting $\mathcal{N}=(0,4)$ dilaton supergravity. Most of this section, as well as section 3 , is not new and presented for the sake of completeness. The superspace formulation of the new

$\mathcal{N}=(0,4)$ dilaton supergravity is presented in section 7 . In the appendix A we present a straightforward but technical world-sheet calculation of string amplitudes corresponding to the target space metric of the effective two-dimensional theory, and in the appendix B we list extra derivative constraints arising from de-gauging $\mathcal{N}=(2,2)$ non-minimal supergravity. Appendix $\mathrm{C}$ contains components of the covariant derivative in $\mathcal{N}=(2,2)$ dilaton supergravity needed in section 5. Finally, in appendix D we repeat the derivation [6] of the chiral density projection formula in $\mathcal{N}=(2,2)$ dilaton supergravity. We begin in the next section with a summary of notations and definitions used throughout the paper.

\section{Calabi-Yau Four-folds: Some Conventions and Definitions}

We study compactification of Type II string theory on $M_{(2)} \times X$ where $M_{(2)}$ is a maximally symmetric homogeneous two-dimensional space-time and $X$ is a Calabi-Yau four-fold. We use the following notations for the space-time indices. Capital letters $M$, $N, \ldots$ run from 0 to 9 and denote ten-dimensional Lorentz indices. Latin letters $m, n$, $\ldots$ and $a, b, \ldots$ represent, respectively, real and holomorphic indices tangent to $X$. Greek 
letters $\alpha, \beta, \ldots$ and $\mu, \nu, \ldots$ are used for the two-dimensional spinor indices "+" and "-", and light-cone indices "₹" and "=", correspondingly. Sometimes we also use capital latin letters $A, B, \ldots$ to denote both spinor and vector indices.

A Calabi-Yau space $X$ is a compact Kähler manifold with complex dimension four and $S U(4)$ holonomy group. It follows that $X$ is a Ricci-flat manifold and, therefore, it can be used as a background for Type II string compactification. As a topological space, $X$ is classified by the Hodge numbers $h^{p, q}$ which count the number of harmonic $(p, q)$-forms $\omega_{i}^{(p, q)} \in H^{p, q}(X), i=1, \ldots h^{p, q}$. The non-vanishing cohomology groups have the following dimensions [7]:

$$
\begin{gathered}
h^{1,1}=h^{3,3}, \quad h^{3,1}=h^{1,3}, \\
h^{2,1}=h^{1,2}=h^{3,2}=h^{2,3}, \\
h^{0,0}=h^{4,4}=h^{4,0}=h^{0,4}=1, \\
h^{2,2}=2\left(22+2 h^{1,1}+2 h^{3,1}-h^{2,1}\right) .
\end{gathered}
$$

For the Euler number of $X$ we have:

$$
\frac{\chi}{6}=8+h^{1,1}+h^{3,1}-h^{2,1}
$$

We denote by $\Omega$ a covariantly constant $(4,0)$-form. The $(1,1)$ - and $(3,1)$-forms are related to the deformation parameters of the Kähler form and the complex structure of $X$, respectively. Namely, an arbitrary variation of the metric of the Calabi-Yau four-fold $X$ that respects $S U(4)$ holonomy looks like:

$$
\delta g_{a \bar{b}} d z^{a} d \bar{z}^{\bar{b}}+\delta g_{a b} d z^{a} d z^{b}+\text { c.c. }
$$

where

$$
\delta g_{a b}=\sum_{j=1}^{h^{3,1}} \phi^{j} w_{a b}^{j}, \quad i \delta g_{a \bar{b}}=\sum_{i=1}^{h^{1,1}} s^{i} \omega_{a \bar{b}}^{i} .
$$

By the appropriate contraction with $\bar{\Omega}$, from the forms $w_{a b}^{j}$ we can construct elements in $H^{1,3}(X)$ :

$$
\omega_{j}^{(1,3)}=\bar{\Omega}_{\bar{a} \bar{b} \bar{c} \bar{d}} \bar{g}^{\bar{d} d} w_{d f}^{j} d \bar{z}^{\bar{a}} d \bar{z}^{\bar{b}} d \bar{z}^{\bar{c}} d z^{f}
$$

In what follows we will use some integrals over the Calabi-Yau space $X$ [8,9]:

$$
\mathcal{V}=\int_{X} d^{8} z \sqrt{g}=\frac{1}{4 !} \int_{X} \mathcal{K} \wedge \mathcal{K} \wedge \mathcal{K} \wedge \mathcal{K}
$$




$$
\begin{gathered}
\mathcal{G}_{\phi_{i} \bar{\phi}_{\bar{j}}}=\frac{1}{4 \mathcal{V}} \int_{X} d^{8} z \sqrt{g} w_{i a b} \bar{w}_{\bar{j}}^{a b} \\
\mathcal{G}_{\sigma_{k} \bar{\sigma}_{l}}=\frac{1}{2 \mathcal{V}} \int_{X} d^{8} z \sqrt{g} \omega_{a \bar{b}}^{k} \omega^{l a \bar{b}} \\
Y_{i m \bar{n}}=\int_{X} \omega_{i}^{(1,1)} \wedge \omega_{m}^{(2,1)} \wedge \omega_{\bar{n}}^{(1,2)} \\
d_{i j k l}=\int_{X} \omega_{i}^{(1,1)} \wedge \omega_{j}^{(1,1)} \wedge \omega_{k}^{(1,1)} \wedge \omega_{l}^{(1,1)}
\end{gathered}
$$

The notations (2.4) and (2.5) will become clear in the next section where we identify expectation values of the fields $s_{i}$ and $\phi_{j}$ with the Kähler and complex structure moduli, respectively. In particular, we write:

$$
\mathcal{K}=i g_{a \bar{b}} d z^{a} \wedge d \bar{z}^{\bar{b}}=\sum_{i=1}^{h^{1,1}}\left\langle s_{i}\right\rangle \omega_{i}^{(1,1)}
$$

for the Kähler form on $X$. The moduli space of a Calabi-Yau space is locally a product of the moduli space of complex deformations, $\mathcal{M}_{c}(X)$, and the (complexified) moduli space

of Kähler structure, $\mathcal{M}_{\mathcal{K}}(X)$. Notice, the metric $\mathcal{G}_{\phi_{i} \bar{\phi}_{\bar{j}}}$ defined above is the Weil-Petersson metric on the moduli space of complex structure of $X$, with the Kähler potential, $c f$. [8]:

$$
K\left(\phi_{i}, \bar{\phi}_{\bar{i}}\right)=-\ln \left(\int_{X} \Omega \wedge \bar{\Omega}\right) .
$$

\section{Compactification of Type IIA String Theory on Calabi-Yau Four-folds}

In this section we describe the effective two-dimensional theory constructed from compactification of Type IIA string theory on a Calabi-Yau four-fold $X$. When the volume of $X$ is large compared to the string scale, Type IIA supergravity is a good low-energy approximation to Type IIA string theory. Therefore, in the 'large volume limit' we may describe the low-energy effective theory studying compactification of Type IIA supergravity on the Calabi-Yau space $X$. With this motivation, let us start this section recalling some facts about Type IIA supergravity itself.

The bosonic field content of Type IIA supergravity contains the metric $g_{M N}$, the dilaton $\varphi$, a vector field $A_{M}$, and tensor fields $B_{M N}$ and $C_{M N P}$. The bosonic part of the Lagrangian (in string frame) looks like:

$$
L_{(10)}=\sqrt{-g}\left[\frac{1}{2} e^{-2 \varphi}\left(R^{(10)}+4(\nabla \varphi)^{2}-\frac{1}{12} H^{2}\right)-\frac{1}{4} F^{2}-\frac{1}{48} G^{2}\right]+\ldots
$$


where we introduced the gauge-invariant field strengths:

$$
\begin{gathered}
F=d A, \quad H=d B, \\
G=d C, \quad G^{\prime}=G+A \wedge H .
\end{gathered}
$$

With this choice of normalization the fields $A, B$, and $C$ transform in a natural, dilatonindependent way under gauge transformations.

The dots in the Lagrangian (3.1) stand for higher order terms among which we find the Chern-Simons term $B \wedge G \wedge G$ and the anomaly term $B \wedge I_{8}$, where the eight-form $I_{8}$ is proportional to the Euler density of $X$. After integration over a compact eight-manifold $X$ these topological terms produce a global anomaly [10,11]:

$$
N=\frac{\chi}{24}-\frac{1}{2(2 \pi)^{2}} \int_{X} G \wedge G
$$

To cancel the tadpole for the $B$-field one has to introduce $N$ fundamental strings filling two-dimensional non-compact space.

The action of Type IIA supergravity is invariant under 16 left and 16 right supersymmetry transformations, such that the left supersymmetries are chiral while the right supersymmetries are anti-chiral with respect to the ten-dimensional chirality operator $\Gamma_{11}$. Since $X$ admits a nowhere vanishing complex spinor of definite chirality, compactification of Type IIA string theory on $X$ is described by $\mathcal{N}=(2,2)$ supergravity theory coupled to matter. With the appropriate choice of orientation, the fundamental strings filling two-dimensional space-time do not break supersymmetry further.

To find the spectrum of the effective low-energy theory we perform Kaluza-Klein reduction of Type IIA supergravity to two dimensions. Below we describe the decomposition of Type IIA bosonic fields in harmonics of $X$. By supersymmetry, incorporation of fermionic zero-modes completes the resulting spectrum into appropriate $\mathcal{N}=(2,2)$ supermultiplets. Via dimensional reduction Type IIA dilaton $\varphi$ becomes a real scalar field in the two-dimensional theory. The ten-dimensional metric $g_{M N}$ decomposes into the twodimensional metric $g_{\mu \nu}, h^{3,1}$ complex scalars $\phi_{i}$ and $h^{1,1}$ real scalars $s_{j}$ defined in (2.3). The antisymmetric tensor fields $B_{M N}$ and $C_{M N P}$ can be expanded into harmonic modes as follows:

$$
B=\sum_{i=1}^{h^{1,1}} r^{i} \omega_{i}^{(1,1)}
$$




$$
C=\sum_{j=1}^{h^{1,1}} A_{\mu}^{j} \omega_{j}^{(1,1)}+\sum_{k=1}^{h^{2,1}} z^{k} \omega_{k}^{(2,1)}+\text { c.c. }
$$

It is convenient to combine real fields $s^{i}$ and $r^{i}$ into complex scalars $\sigma^{i}$. Taking into account the vector field $A_{\mu}$ from the Ramond-Ramond sector of Type IIA theory, we end up with the following list of $\mathcal{N}=(2,2)$ supermultiplets:

$$
\begin{gathered}
\text { a gravitational multiplet: } g_{\mu \nu}, \quad A_{\mu}, \quad \varphi \\
h^{3,1} \text { chiral multiplets : } \phi_{i}, \quad \bar{\phi}_{\bar{i}} \\
h^{1,1} \text { twisted chiral multiplets : } \sigma^{j}, \quad \bar{\sigma}^{j}, \quad A_{\mu}^{j} \\
h^{2,1} \text { (twisted) chiral multiplets : } z^{k}, \quad \bar{z}^{\bar{k}}
\end{gathered}
$$

Vector fields $A_{\mu}$ and $A_{\mu}^{i}$ do not have propagating degrees of freedom in two dimensions and play the role of auxiliary fields in the supergravity multiplet and twisted chiral multiplets, respectively.

The complex scalar fields $z^{k}$ which come from $(2,1)$-modes take value in a torus. When background fluxes satisfy $G \wedge \omega_{k}^{(2,1)}=0$ and $H \wedge^{*} \omega_{k}^{(2,1)}=0$ there is no superpotential for the corresponding harmonics $z_{k}$, so these fields are massless. A $T$-duality transformation on the torus then converts them from ordinary chiral superfields to twisted chiral superfields. It is natural to choose $z_{k}$ to be scalar components of chiral superfields. Indeed, if we start in eleven dimensions, the reduction of the $C$-field (3.4) yields $h^{2,1}$ complex scalar modes in three dimensions. These modes are scalar components of chiral superfields since there is no notion of "twisted chiral superfields" in three dimensions. After a further compactification on a circle they naturally remain as chiral superfields in two dimensions, but now a $T$ duality becomes possible and $z_{k}$ can be alternatively described if one prefers as twisted chiral superfields.

To find the effective action for the light fields we have to substitute (2.3), (3.3) and (3.4) in the Lagrangian (3.1) and integrate over the internal space $X$. Using the formulas (2.4), (2.5) and (2.6) we obtain the following effective action for the bosonic modes, $c f$. [9,5]:

$$
\begin{gathered}
L_{(2)}=e^{-2 \varphi} \mathcal{V}\left[R^{(2)}+4(\nabla \varphi)^{2}-\mathcal{G}_{\phi_{i} \bar{\phi}_{\bar{j}}}\left(\partial_{\mu} \phi^{i}\right)\left(\partial^{\mu} \bar{\phi}^{\bar{j}}\right)-\right. \\
\left.-\frac{1}{2} \mathcal{G}_{\sigma_{i} \bar{\sigma}_{j}}\left(\partial_{\mu} \sigma^{i}\right)\left(\partial_{\mu} \bar{\sigma}^{j}\right)\right]-\frac{1}{4} Y_{i m \bar{n}} \sigma^{i}\left(D_{\mu} z^{m}\right)\left(D^{\mu} \bar{z}^{\bar{n}}\right)+\ldots
\end{gathered}
$$


where the covariant derivative $D_{\mu}$ acting on $z^{m}$ contains a connection corresponding to the holomorphic dependence of the basis of $(2,1)$-forms on the complex structure [9].

To summarize, we find that in the large volume limit compactification of Type IIA string theory on a Calabi-Yau four-fold $X$ leads to $\mathcal{N}=(2,2)$ dilaton supergravity coupled to a non-linear sigma-model. The target space of this sigma-model is parametrized by some number of chiral and twisted chiral multiplets. This agrees with the result of [12], where it was found that the most general $\mathcal{N}=(2,2)$ non-linear sigma-model is based on a target space with two non-commuting complex structures $J_{ \pm}$, so that the space $\operatorname{ker}\left(J_{+}-J_{-}\right)$is parametrized by chiral superfields, while $\operatorname{ker}\left(J_{+}+J_{-}\right)$is parametrized by twisted chiral superfields.

This kind of dilaton supergravity coupled to $\mathcal{N}=(2,2)$ chiral matter was studied some time ago [5]. However, for our purposes we need to generalize the component construction of [5] to include twisted chiral multiplets. Furthermore, background fluxes of Ramond-Ramond field strengths induce effective superpotential [13, 14] and/or twisted chiral superpotential [13, 14, 15] in the two-dimensional theory. Hence, we have to incorporate these terms in the construction as well. The most elegant and convenient way to do this is in $\mathcal{N}=(2,2)$ superspace where the supersymmetry becomes manifest [16, 17]. In addition to the usual space-time coordinates $x^{\mu}, \mathcal{N}=(2,2)$ superspace is parametrized by anti-commuting coordinates $\theta^{\alpha}=\left(\theta^{+}, \theta^{-}\right)$and their complex conjugates $\bar{\theta}^{\dot{\alpha}}=\left(\bar{\theta}^{\dot{+}}, \bar{\theta}^{\dot{-}}\right)$. Then, we expect that the action of the matter fields (3.5) can be written in a compact form, similar to the action of matter coupled $\mathcal{N}=1$ supergravity in four dimensions:

$$
S=\int d^{2} x \int d^{2} \theta d^{2} \bar{\theta} E^{-1} \exp (-K)
$$

We postpone the discussion of the superspace measure $E$ till the next sections where superspace formulation will be discussed in detail. Now we simply assume that the suitable measure exists. The main advantage of the superspace formulation is that due to the extended supersymmetry, all the term in the action (3.5) with up to two derivatives or four fermions are determined by a single real function $K\left(\phi_{i}, \bar{\phi}_{\bar{i}}, \sigma_{j}, \bar{\sigma}_{j}, z_{k}, \bar{z}_{\bar{k}}, \varphi\right)$, the Kähler potential [18]. It is invariant under the generalized Kähler transformation:

$$
K \longrightarrow K+\Lambda_{1}\left(\phi_{i}, \sigma_{j}, z_{k}\right)+\bar{\Lambda}_{1}\left(\bar{\phi}_{\bar{i}}, \bar{\sigma}_{j}, \bar{z}_{\bar{k}}\right)+\Lambda_{2}\left(\phi_{i}, \bar{\sigma}_{j}, \bar{z}_{\bar{k}}\right)+\bar{\Lambda}_{2}\left(\bar{\phi}_{\bar{i}}, \sigma_{j}, z_{k}\right)
$$

The target space metric is given by the second derivative of the Kähler potential. 
For the sake of simplicity, let us assume for a moment that $h^{2,1}=0$. Then the metric is block diagonal:

$$
\mathcal{G}_{\phi_{i} \bar{\sigma}_{j}}=\frac{\partial^{2} K}{\partial \phi_{i} \partial \bar{\sigma}_{j}}=0
$$

From the condition (3.8) it follows that locally we can write the Kähler potential that gives the effective action (3.5) as:

$$
K=K_{c}\left(\phi_{i}, \bar{\phi}_{\bar{i}}\right)+K_{\mathcal{K}}\left(\sigma_{j}, \bar{\sigma}_{j}\right)
$$

where $K_{c}$ is the Kähler potential (2.9) on the moduli space of the complex structure:

$$
K_{c}\left(\phi_{i}, \bar{\phi}_{\bar{i}}\right)=-\ln \left(\int_{X} \Omega \wedge \bar{\Omega}\right)
$$

Similar to the case of Calabi-Yau three-folds [8], one can verify that the metric $\mathcal{G}_{\sigma_{i}} \bar{\sigma}_{j}$ can be obtained from the Kähler potential:

$$
K_{\mathcal{K}}\left(\sigma_{j}, \bar{\sigma}_{j}\right)=-\ln \left(\int_{X} \mathcal{K} \wedge \mathcal{K} \wedge \mathcal{K} \wedge \mathcal{K}\right)
$$

Indeed, if $\omega^{(2)}$ is a harmonic 2-form on a Calabi-Yau four-fold $X$, its Hodge dual is given by the following neat formula:

$$
{ }^{*} \omega^{(2)}=-\frac{1}{2} \omega^{(2)} \wedge \mathcal{K} \wedge \mathcal{K}+\frac{2}{3} \frac{\left(\int_{X} \omega^{(2)} \wedge \mathcal{K} \wedge \mathcal{K} \wedge \mathcal{K}\right)}{\left(\int_{X} \mathcal{K} \wedge \mathcal{K} \wedge \mathcal{K} \wedge \mathcal{K}\right)} \mathcal{K} \wedge \mathcal{K} \wedge \mathcal{K}
$$

Therefore, we can write (2.5) in the following form:

$$
\begin{gathered}
\mathcal{G}_{\sigma_{k} \bar{\sigma}_{l}}=-\frac{1}{2 \mathcal{V}} \int_{X} \omega_{k}^{(1,1)} \wedge^{*} \omega_{l}^{(1,1)}= \\
=\frac{1}{4 \mathcal{V}} \int_{X} \omega_{k}^{(1,1)} \wedge \omega_{l}^{(1,1)} \wedge \mathcal{K} \wedge \mathcal{K}-\frac{1}{72 \mathcal{V}^{2}}\left(\int_{X} \omega_{k}^{(1,1)} \wedge \mathcal{K} \wedge \mathcal{K} \wedge \mathcal{K}\right)\left(\int_{X} \omega_{l}^{(1,1)} \wedge \mathcal{K} \wedge \mathcal{K} \wedge \mathcal{K}\right)
\end{gathered}
$$

Using the explicit expression (2.8) for the Kähler form $\mathcal{K}$, it is easy to see that the above metric indeed follows from the Kähler potential (3.10):

$$
\mathcal{G}_{\sigma_{k} \bar{\sigma}_{l}}=-\frac{1}{2} \frac{\partial^{2} K_{\mathcal{K}}\left(\sigma_{j}, \bar{\sigma}_{j}\right)}{\partial \sigma_{k} \partial \bar{\sigma}_{l}}
$$

Hence, to the leading order the metric on the target space is Kähler, torsionless, and equal to the metric on the moduli space of the Calabi-Yau space $X, \mathcal{M}_{c}(X) \times \mathcal{M}_{\mathcal{K}}(X)$. 
The classical action (3.5) is invariant under two $U(1) R$-symmetries. We will denote their linear combinations as $U(1)_{A}$ and $U(1)_{V}$. The action of these symmetries on the supercharges can be represented as:

$$
\begin{array}{ll}
Q_{-} & \bar{Q}_{\dot{+}} \\
Q_{+} & \bar{Q} \dot{-}
\end{array}
$$

where the upper (lower) row is assigned a $U(1)_{A}$ charge $+1(-1)$ while the right (left) column is assigned a $U(1)_{V}$ charge $+1(-1)$. These R-symmetries are not symmetries of the string theory - there is no way to assign $R$-transformations to massive string modes to preserve them. Even though we will not explicitly include massive string modes in the present paper, we will include a superpotential and twisted chiral superpotential that violate the $R$-symmetries. Even in the absence of the superpotentials, higher derivative interactions among the massless fields obtained by integrating out massive string states would be expected to violate the $R$-symmetry.

The explicit expression for the chiral superpotentials generated by the most general Ramond-Ramond flux $\mathcal{F}=\sum(R R$ fields $)$ in terms of the Calabi-Yau moduli was derived in [14]:

$$
W\left(\phi_{i}\right)=\frac{1}{2 \pi} \int_{X} \Omega \wedge G
$$

and for the twisted chiral superpotential:

$$
\widetilde{W}\left(\sigma_{j}\right)=\frac{1}{2 \pi} \int_{X} e^{\mathcal{K}} \wedge \mathcal{F}
$$

The superpotential $W\left(\phi_{i}\right)$ and the twisted superpotential $\widetilde{W}\left(\sigma_{j}\right)$ are holomorphic functions of the fields $\phi_{i}$ and $\sigma_{j}$, respectively. Taking into account the superpotential terms, the action of the matter fields reads as:

$$
\begin{gathered}
S_{(2)}=\int d^{2} x \int d^{2} \theta d^{2} \bar{\theta} E^{-1} e^{-K}+\int d^{2} x \int d^{2} \theta \mathcal{E}^{-1} W\left(\phi_{i}\right)+ \\
+\int d^{2} x \int d \theta^{+} d \theta^{-} \widetilde{\mathcal{E}}^{-1} \widetilde{W}\left(\sigma_{j}\right)+\text { c.c. }
\end{gathered}
$$


Generic values of Ramond-Ramond fluxes completely break the $\mathcal{N}=(2,2)$ supersymmetry 3 . In the two-dimensional theory this effect corresponds to generation of a superpotential that lifts (part of) supersymmetric vacua. However, if the vacuum values of the fields $\phi_{i}$ and $\sigma_{j}$ satisfy:

$$
\frac{D W}{D \phi_{i}}=0 \quad \text { and } \quad \frac{D \widetilde{W}}{D \sigma_{i}}=0
$$

then Type IIA compactification on the corresponding Calabi-Yau manifold is supersymmetric [13,14]. From the formulas (2.2), (3.2) and the quantization condition of the $G$-flux 111] it follows that there is a finite number of choices for $[G] \in H^{4}(X)$ corresponding to supersymmetric vacua. In particular, if $h^{2,1}>8+h^{1,1}+h^{3,1}$, then there are no such vacua at all.

From the superspace construction in section 5 it follows that in the equations (3.16) we should use the appropriate covariant derivatives:

$$
\frac{D W}{D \phi_{i}}=\frac{\partial W}{\partial \phi_{i}}+\frac{\partial K_{c}\left(\phi_{i}, \bar{\phi}_{\bar{i}}\right)}{\partial \phi_{i}} W, \quad \frac{D \widetilde{W}}{D \sigma_{i}}=\frac{\partial \widetilde{W}}{\partial \sigma_{i}}+\frac{\partial K_{\mathcal{K}}\left(\sigma_{j}, \bar{\sigma}_{j}\right)}{\partial \sigma_{i}} \widetilde{W}
$$

where $K_{c}$ and $K_{\mathcal{K}}$ are given by the tree-level formulas (2.9) and (3.10), respectively. A simple way to see that one has to use the covariant derivatives instead of ordinary ones is to consider first compactification of F-theory on the same Calabi-Yau space $X$. In the component action of the effective $\mathcal{N}=1$ four-dimensional theory there is a scalar potential:

$$
e^{K}\left(\mathcal{G}^{\phi_{i} \bar{\phi}_{\bar{j}}}\left(D_{\phi_{i}} W\right)\left(D_{\bar{\phi}_{\bar{j}}} \bar{W}\right)-3|W|^{2}\right)
$$

where the covariant derivative $D_{\phi_{i}} W=\frac{D W}{D \phi_{i}}$ is defined in (3.17). After a further compactification on a torus $T^{2}$, this theory is dual to compactification of Type IIA string theory

3 Investigating the supersymmetry conditions as in [13,14, 19], one can also show that any $H$ field flux breaks all the supersymmetry. A simple way to see this is to assume, on the contrary, that there exists a supersymmetric vacuum corresponding to a non-zero $H$-flux and consider a BPS soliton connecting such a vacuum to the vacuum with zero $H$-flux. In Type IIA string theory this soliton would correspond to an NS5-brane wrapped over a Poincaré dual supersymmetric 5-cycle. However, there is a contradiction since Calabi-Yau 4-folds do not have supersymmetric 5-cycles, see e.g. [14]. Therefore, a non-zero $H$-flux lifts all the supersymmetric vacua. It is natural to interpret this in terms of the effective superpotential $W \sim \int C \wedge^{*} H$ for the scalar fields $z_{k}$.

4 Of course, here we assume that $X$ is elliptically fibered. The result, however, is independent of this assumption. 
on $X$. It is clear that after the dimensional reduction of the four-dimensional component action the covariant derivatives (3.17) also appear in the component action of the two-dimensional theory in question.

These models can have a variety of $T$-duality symmetries. Of particular interest are mirror symmetries [20,21]. A mirror symmetry is, of course, a symmetry that maps Type IIA string theory on a four-fold $X$ to Type IIA string theory on the mirror variety $\widetilde{X}$, such that:

$$
h^{p, q}(X)=h^{4-p, q}(\widetilde{X})
$$

and the conformal field theories associated with $X$ and $\widetilde{X}$ are equivalent. This operation corresponds [4] to a transformation which exchanges chiral multiplets and twisted chiral multiplets. It can be interpreted [22] in terms of the supergeometrical coordinate transformation $\theta^{-} \leftrightarrow \theta^{-}$that also exchanges chiral multiplets and twisted chiral multiplets and changes the superspace measure in a way consistent with other definitions of mirror symmetry. In particular, the latter implies that under the mirror symmetry we have $\sigma_{i} \leftrightarrow \phi_{j}$ which is consistent with our interpretation of (vevs of) these fields as the Kähler and the complex structure moduli of the Calabi-Yau space $X$. Therefore, the mirror symmetry relates different quantum $\mathcal{N}=(2,2)$ theories also interchanging:

$$
\begin{aligned}
U(1)_{A} & \leftrightarrow U(1)_{V} \\
\phi_{i} & \leftrightarrow \sigma_{j} \\
W\left(\phi_{i}\right) & \leftrightarrow \widetilde{W}\left(\sigma_{j}\right)
\end{aligned}
$$

The mirror map has no effect on the $\mathcal{N}=(2,2)$ dilaton supergravity itself, so that in the absence of matter fields it must be mirror-symmetric. It may seem that twisted chiral fields $z_{k}$ violate the invariance under (3.20). Recall that via a spacetime T-duality transformation, those fields can be described by either chiral or twisted chiral superfields. Hence mirror symmetry just exchanges these two descriptions.

By definition, the low-energy effective action (3.15) describes dynamics of the light modes in Type IIA string theory on $X$ in the large volume limit. In other words, tree-level amplitudes in Type IIA string theory must agree with the corresponding amplitudes in the effective two-dimensional theory. In the appendix A we illustrate this by a world-sheet calculation which independently proves that the target space metric is block-diagonal, $c f$. (3.8). 
A superspace formulation of $\mathcal{N}=(2,2)$ dilaton supergravity that includes chiral and twisted chiral multiplets on equal footing does not seem to exist in the literature. Although a superspace model of $\mathcal{N}=(2,2)$ supergravity where the dilaton is a complex field was constructed in [23], we are interested in a theory where the supergravity multiplet contains a real dilaton field. A superspace formulation of such a supergravity theory is presented in the next section.

\section{Superspace Formulation of $\mathcal{N}=(2,2)$ Dilaton Supergravity}

In this section we present a superspace construction of $\mathcal{N}=(2,2)$ dilaton supergravity without gauged symmetry. This last property is a distinguishing feature of the new formulation since all the known $\mathcal{N}=(2,2)$ gravity theories have at least one gauged $U(1)$ $R$-symmetry (see [22] for a general presentation). Theories where the entire $U(1)_{A} \otimes U(1)_{V}$ symmetry group is gauged are called non-minimal (or reducible), as opposed to minimal theories where only $U(1)_{A}$ or $U(1)_{V}$ factor is gauged. It is very well known how to obtain one supergravity theory with a smaller holonomy group from a supergravity theory with a larger holonomy group. This process has been for a long time called "de-gauging" (see [16], section 5.3.b.7).

The basic idea of de-gauging is to break the gauge symmetry introducing extra matter field in a Goldstone-like mechanism. For example, consider an abelian vector multiplet in a four-dimensional $\mathcal{N}=1$ gauge theory. It contains a $U(1)$ gauge vector field, gaugino and an auxiliary field. All the other fields can be set to zero by a supersymmetric choice of gauge, the so-called Wess-Zumino gauge. On the other hand, a massive gauge multiplet contains some extra component fields which could be eliminated in the massless multiplet. The reason is that the massive system no longer possess the $U(1)$ gauge invariance. This toy model teaches us that when a symmetry is broken in superspace, extra component fields not present in the gauge symmetric phase begin to appear. In other words, Goldstone supermultiplets must appear. And their component fields come from that part of the vector multiplet that was ignored in the symmetric phase. Following these steps, we construct $\mathcal{N}=(2,2)$ dilaton supergravity via de-gauging $U(1)_{A} \otimes U(1)_{V}$ non-minimal gauged supergravity. An advantage of this approach is that both the original and the resulting theories are manifestly invariant under the mirror symmetry (3.20). We also find that the new $\mathcal{N}=(2,2)$ supergravity multiplet contains a real dilaton field $\varphi$, in accordance with the results of section 3 where we studied compactification of Type IIA 
string theory on Calabi-Yau four-folds. We hope that apart from this obvious application there may also be many other aspects of the new supergravity to explore. For example, it would be interesting to study black hole solutions in this dilaton supergravity, $c f$. [24].

The relation between different supergravity theories can be schematically represented in the form of the following diagram:

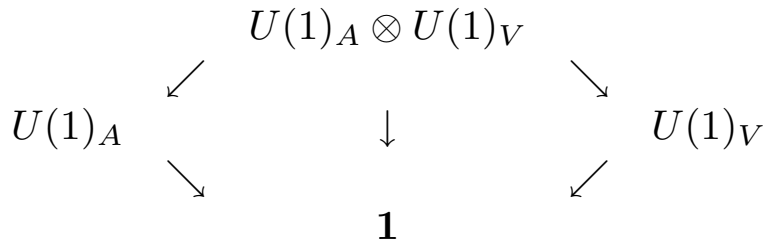

where the theory with the trivial holonomy group is the new $\mathcal{N}=(2,2)$ dilaton supergravity we are going to construct. Notice, however, that various arrows in this diagram have different meaning. For example, minimal theories with either of the $U(1)$ R-symmeties gauged can be obtained by truncation of the non-minimal $U(1)_{A} \otimes U(1)_{V}$ gauged supergravity [22]. On the other hand, the vertical arrow corresponds to de-gauging $U(1)_{A} \otimes U(1)_{V}$ symmetry, so that the total number of degrees of freedom increases. More precisely, it has to be a combination of consistent truncation of the non-minimal $\mathcal{N}=(2,2)$ supergravity to a minimal one plus a de-gauging of the latter. To see this, let us count the number of real Goldstone scalars. Since the broken $U(1)_{A} \otimes U(1)_{V}$ phase of the theory has exactly the same field content as the gauge symmetric phase plus the field content of the Goldstone multiplets minus the parts that go into the longitudinal components of the $U(1)_{A} \otimes U(1)_{V}$ gauge fields, we find that in total Goldstone multiplets should have three real scalars. However, there are no mirror-symmetric $\mathcal{N}=(2,2)$ multiplets with such a field content. Therefore, we conclude that the vertical arrow should be a more economical de-gauging. Indeed, if we were following another route via a minimal gauged supergravity, at the first step we would have to make a consistent truncation that would eliminate one of the gauge fields. In order to de-gauge the resulting minimal gauged supergravity we would have to introduce extra chiral (or twisted chiral) Goldstone superfield. In any case, one real scalar from this multiplet would become the longitudinal component of the gauge vector field, and the other would become a dilaton, in agreement with what we expect. Assuming that the latter route (which is not, unfortunately, mirror-symmetric) is equivalent to the vertical arrow on the above diagram, we expect that there is an economical de-gauging of the non-minimal $\mathcal{N}=(2,2)$ supergravity that leads to only one massless scalar (the dilaton). 
To start the construction, let us arrange component fields found in the previous section into superfields. First we give the definitions in flat superspace and then extend them to curved superspace. Left-right symmetric $\mathcal{N}=2$ superspace is parametrized by the bosonic coordinates $x^{\mu}=\left(x^{\ddagger}, x^{=}\right)$, two anti-commuting complex spinor coordinates $\theta^{\alpha}=\left(\theta^{+}, \theta^{-}\right)$ and their complex conjugates $\bar{\theta}^{\dot{\alpha}}=\left(\bar{\theta}^{\dot{+}}, \bar{\theta}^{\dot{-}}\right)$. The spinor derivatives $D_{+}, D_{-}, D_{\dot{+}}$ and $D_{-}$ satisfy $\left\{D_{+}, D_{\dot{+}}\right\}=\partial_{\neq}$and $\left\{D_{-}, D_{\dot{-}}\right\}=\partial_{=}$, with all other (anti-)commutators vanishing.

Irreducible matter superfields are defined by imposing some constraints on general complex superfields. The simplest constraints are linear in derivatives and look like:

$$
D_{\dot{+}} \Phi=D_{-} \dot{ } \Phi=D_{+} \bar{\Phi}=D_{-} \bar{\Phi}=0
$$

for a chiral superfield $\Phi$, and:

$$
D_{\dot{+}} \Sigma=D_{-} \Sigma=D_{+} \bar{\Sigma}=D_{\dot{-}} \bar{\Sigma}=0
$$

for a twisted chiral superfield $\Sigma$.

In what follows we promote the complex scalar fields $\phi_{i}$ and $\sigma_{j}$ defined in the previous section to the chiral and twisted chiral superfields $\Phi_{i}$ and $\Sigma_{j}$, respectively. Similarly, we will regard the compact fields $z_{k}$ as the scalar components of (twisted) chiral superfields $\mathcal{Z}_{k}$.

Components of the chiral superfield $\Phi_{i}$ can be obtained using the projection method:

$$
\begin{array}{rlrl}
\Phi_{i} \mid & =\phi_{i}, & \bar{\Phi}_{i} \mid=\bar{\phi}_{i} \\
D_{+} \Phi_{i} \mid & =\psi_{+}^{i}, & & D_{\dot{+}} \bar{\Phi}_{i} \mid=\psi_{\dot{+}}^{i} \\
D_{-} \Phi_{i} \mid & =\psi_{-}^{i}, & D_{\dot{-}} \bar{\Phi}_{i} \mid=\psi_{\dot{-}}^{i} \\
\frac{i}{2}\left[D_{+}, D_{-}\right] \Phi_{i} \mid & =A_{i}, & & \frac{i}{2}\left[D_{\dot{+}}, D_{-}\right] \bar{\Phi}_{i} \mid=\bar{A}_{i}
\end{array}
$$

where, for example, $\Phi_{i} \mid$ denotes the leading component of the superfield $\Phi_{i}$, with all the $\theta$-coordinates put to zero. Similarly, we find the components of a twisted chiral multiplet $\Sigma_{j}$ :

$$
\begin{aligned}
& \Sigma_{j}\left|=\sigma_{j}, \quad \bar{\Sigma}_{j}\right|=\bar{\sigma}_{j} \\
& D_{+} \Sigma_{j}\left|=\zeta_{+}^{j}, \quad D_{\dot{+}} \bar{\Sigma}_{j}\right|=\zeta_{\dot{+}}^{j} \\
& D_{-} \Sigma_{j}\left|=\zeta_{\dot{-}}^{j}, \quad D_{-} \bar{\Sigma}_{j}\right|=\zeta_{-}^{j}
\end{aligned}
$$




$$
\frac{i}{2}\left[D_{+}, D_{-}\right] \Sigma_{j}\left|=B_{j}, \quad \frac{i}{2}\left[D_{\dot{+}}, D_{-}\right] \bar{\Sigma}_{j}\right|=\bar{B}_{j}
$$

To define chiral and twisted chiral superfields in curved $\mathcal{N}=(2,2)$ superspace, the spinor derivatives $D_{\alpha}$ must be appropriately replaced by the covariant derivatives $\nabla_{\alpha}$.

In our construction of $\mathcal{N}=(2,2)$ dilaton supergravity we start with non-minimal gauged supergravity and then de-gauge $U(1)_{A} \otimes U(1)_{V}$ symmetry. If we introduce superfields $\mathcal{A}_{\alpha}$ and $\mathcal{A}_{\alpha}^{\prime}$ representing $U(1)_{V}$ and $U(1)_{A}$ gauge connections, and denote by $\Lambda_{\alpha}$ the Lorentz spin-connection, then the covariant derivative in this theory has the form:

$$
\nabla_{\alpha}=E_{\alpha}^{B} D_{B}+\Lambda_{\alpha} \mathcal{X}+\mathcal{A}_{\alpha} \mathcal{Y}+\mathcal{A}_{\alpha}^{\prime} \mathcal{Y}^{\prime}
$$

where $E_{\alpha}{ }^{B}$ is the supervielbein.

The Lorentz generators, $\mathcal{X}, U(1)_{V}$ symmetry generators, $\mathcal{Y}$, and $U(1)_{A}$ symmetry generators, $\mathcal{Y}^{\prime}$, act on the covariant derivative $\nabla_{\alpha}$ in the following way [22]:

$$
\begin{aligned}
& {\left[\mathcal{X}, \nabla_{ \pm}\right]= \pm \frac{1}{2} \nabla_{ \pm} \quad, \quad\left[\mathcal{X}, \nabla_{ \pm}\right]= \pm \frac{1}{2} \nabla_{ \pm}} \\
& {\left[\mathcal{Y}, \nabla_{ \pm}\right]=-\frac{i}{2} \nabla_{ \pm} \quad, \quad\left[\mathcal{Y}, \nabla_{ \pm}\right]=+\frac{i}{2} \nabla_{ \pm}} \\
& {\left[\mathcal{Y}^{\prime}, \nabla_{ \pm}\right]=\mp \frac{i}{2} \nabla_{ \pm} \quad, \quad\left[\mathcal{Y}^{\prime}, \nabla_{ \pm}\right]= \pm \frac{i}{2} \nabla_{\dot{ \pm}}}
\end{aligned}
$$

The constraints which define non-minimal $\mathcal{N}=2 U(1)_{A} \otimes U(1)_{V}$ supergravity are given by:

$$
\begin{gathered}
\left\{\nabla_{+}, \nabla_{+}\right\}=0, \quad\left\{\nabla_{-}, \nabla_{-}\right\}=0 \\
\left\{\nabla_{+}, \nabla_{\dot{+}}\right\}=i \nabla_{\ddagger}, \quad\left\{\nabla_{-}, \nabla_{\dot{-}}\right\}=i \nabla_{=} \\
\left\{\nabla_{+}, \nabla_{-}\right\}=-\frac{1}{2} \bar{R}\left(\overline{\mathcal{X}}-i \overline{\mathcal{Y}}^{\prime}\right), \quad\left\{\nabla_{+}, \nabla_{\dot{-}}\right\}=-\frac{1}{2} \bar{F}(\overline{\mathcal{X}}-i \overline{\mathcal{Y}})
\end{gathered}
$$

where the chiral superfield $R$ and the twisted chiral superfield $F$ are related to the twodimensional curvature $R^{(2)}$ and the abelian field strengths of the graviphoton gauge fields. The easiest way to see this is to compute the commutator [22,25]:

$$
\begin{aligned}
{\left[\nabla_{\ddagger}, \nabla_{=}\right] } & =\frac{1}{2}\left(\left(\nabla^{2} R\right)-\frac{1}{2} R \bar{R}+\left(\nabla_{+} \nabla_{\dot{-}} F\right)-\frac{1}{2} F \bar{F}\right) \mathcal{X}+ \\
+ & \frac{i}{2}\left(\nabla_{+} \nabla_{\dot{-}} F\right) \mathcal{Y}+\frac{i}{2}\left(\nabla^{2} R\right) \mathcal{Y}^{\prime}+\ldots+\text { c.c. }
\end{aligned}
$$

where the dots stand for the covariant derivative terms like $\left(\nabla_{-} F\right) \nabla_{\dot{+}}$, etc. If we set $F=0$ in the constraints $(4.8)$, we obtain $U(1)_{A}$ minimal gauged supergravity theory. On 
the other hand, if we set $R=0$, we end up with $U(1)_{V}$ minimal theory. We denote the leading components of the superfields $F$ and $R$ as follows:

$$
F|=G, \quad R|=H
$$

In order to obtain $\mathcal{N}=(2,2)$ supergravity theory without gauged symmetry, we replace the covariant derivative $\nabla_{\alpha}$ by $\widehat{\nabla}_{\alpha}$ which includes only derivatives and Lorentz generator, so that:

$$
\nabla_{\alpha}=\widehat{\nabla}_{\alpha}+\mathcal{A}_{\alpha} \mathcal{Y}+\mathcal{A}_{\alpha}^{\prime} \mathcal{Y}^{\prime}
$$

and the remaining derivatives are real. The new covariant derivative $\widehat{\nabla}_{\alpha}$ contains neither the $U(1)_{V}$ gauge connection nor the $U(1)_{A}$ gauge connection, so it can describe twodimensional $\mathcal{N}=(2,2)$ supergravity without gauged symmetry.

Substituting (4.11) into (4.8), and using (4.7), we derive the form of the commutator algebra for the $\widehat{\nabla}_{\alpha}$ operators:

$$
\begin{gathered}
\left\{\widehat{\nabla}_{+}, \widehat{\nabla}_{+}\right\}=i\left(\lambda_{+}+\widetilde{\lambda}_{+}\right) \widehat{\nabla}_{+} \quad, \quad\left\{\widehat{\nabla}_{-}, \widehat{\nabla}_{-}\right\}=i\left(\lambda_{-}-\widetilde{\lambda}_{-}\right) \widehat{\nabla}_{-} \\
\left\{\widehat{\nabla}_{+}, \widehat{\nabla}_{-}\right\}=-\frac{1}{2} \bar{R} \mathcal{X}+\frac{i}{2}\left(\lambda_{-}+\widetilde{\lambda}_{-}\right) \widehat{\nabla}_{+}+\frac{i}{2}\left(\lambda_{+}-\widetilde{\lambda}_{+}\right) \widehat{\nabla}_{-} \\
\left\{\widehat{\nabla}_{+}, \widehat{\nabla}_{\dot{-}}\right\}=-\frac{1}{2} \bar{F} \mathcal{X}+\frac{i}{2}\left(\lambda_{\dot{-}}+\widetilde{\lambda}_{\dot{-}}\right) \widehat{\nabla}_{+}-\frac{i}{2}\left(\lambda_{+}-\widetilde{\lambda}_{+}\right) \widehat{\nabla}_{\dot{-}} \\
\left\{\widehat{\nabla}_{+}, \widehat{\nabla}_{\dot{+}}\right\}=i \widehat{\nabla}_{+}+\frac{i}{2}\left(\lambda_{\dot{+}}+\widetilde{\lambda}_{\dot{+}}\right) \widehat{\nabla}_{+}-\frac{i}{2}\left(\lambda_{+}+\widetilde{\lambda}_{+}\right) \widehat{\nabla}_{\dot{+}} \\
\left\{\widehat{\nabla}_{-}, \widehat{\nabla}_{\dot{-}}\right\}=i \widehat{\nabla}_{=}+\frac{i}{2}\left(\lambda_{\dot{-}}-\widetilde{\lambda}_{\dot{-}}\right) \widehat{\nabla}_{-}-\frac{i}{2}\left(\lambda_{-}-\widetilde{\lambda}_{-}\right) \widehat{\nabla}_{\dot{-}}
\end{gathered}
$$

where the new fields $\lambda_{\alpha}$ and $\widetilde{\lambda}_{\alpha}$ have appeared. They are components of the non-minimal gauged supergravity multiplet that could be eliminated by a gauge transformation in the $U(1)_{A} \otimes U(1)_{V}$ gauge-symmetric phase. In the theory we are constructing the $U(1)_{A} \otimes$ $U(1)_{V}$ gauge symmetry is broken, so that the fields $\lambda_{\alpha}$ and $\widetilde{\lambda}_{\alpha}$ are dynamical. They have to be identified with the spinorial derivatives of a new matter Goldstone multiplet. To the leading order in $\theta^{\alpha}$, the fields $\lambda_{\alpha}$ and $\widetilde{\lambda}_{\alpha}$ can also be identified with the dilatino field of the new supergravity multiplet.

If we introduce four linear independent spinors (along with their conjugates):

$$
\begin{aligned}
& \eta_{+} \equiv \lambda_{+}+\widetilde{\lambda}_{+}, \quad \eta_{-} \equiv \lambda_{-}-\widetilde{\lambda}_{-} \\
& \widetilde{\eta}_{+} \equiv \lambda_{+}-\widetilde{\lambda}_{+}, \quad \widetilde{\eta}_{-} \equiv \lambda_{-}+\widetilde{\lambda}_{-}
\end{aligned}
$$


then the conditions (4.12) which define $\mathcal{N}=(2,2)$ dilaton supergravity can be written in the following simple form:

$$
\begin{gathered}
\left\{\widehat{\nabla}_{+}, \widehat{\nabla}_{+}\right\}=i \eta_{+} \widehat{\nabla}_{+}, \quad\left\{\widehat{\nabla}_{-}, \widehat{\nabla}_{-}\right\}=i \eta_{-} \widehat{\nabla}_{-} \\
\left\{\widehat{\nabla}_{+}, \widehat{\nabla}_{-}\right\}=-\frac{1}{2} \bar{R} \mathcal{X}+\frac{i}{2} \widetilde{\eta}_{-} \widehat{\nabla}_{+}+\frac{i}{2} \widetilde{\eta}_{+} \widehat{\nabla}_{-} \\
\left\{\widehat{\nabla}_{+}, \widehat{\nabla}_{\dot{-}}\right\}=-\frac{1}{2} \bar{F} \mathcal{X}+\frac{i}{2} \widetilde{\eta}_{\dot{-}} \widehat{\nabla}_{+}-\frac{i}{2} \widetilde{\eta}_{+} \widehat{\nabla}_{\dot{-}} \\
\left\{\widehat{\nabla}_{+}, \widehat{\nabla}_{\dot{+}}\right\}=i \widehat{\nabla}_{\neq}+\frac{i}{2} \eta_{\dot{+}} \widehat{\nabla}_{+}-\frac{i}{2} \eta_{+} \widehat{\nabla}_{\dot{+}} \\
\left\{\widehat{\nabla}_{-}, \widehat{\nabla}_{\dot{-}}\right\}=i \widehat{\nabla}_{=}+\frac{i}{2} \eta_{\dot{-}} \widehat{\nabla}_{-}-\frac{i}{2} \eta_{-} \widehat{\nabla}_{\dot{-}}
\end{gathered}
$$

Note that these equations, as well as the conditions (4.12), are manifestly invariant under the mirror symmetry transformation (3.20).

To summarize, there exists a unique mirror-symmetric two-dimensional $\mathcal{N}=(2,2)$ dilaton supergravity defined by the set of constraints (4.12) on the covariant derivative $\widehat{\nabla}_{\alpha}$. The new supergravity theory does not have gauged symmetry and contains a real dilaton field $\varphi$, as we will show in a moment.

Since we define new $\mathcal{N}=(2,2)$ dilaton supergravity theory imposing constraints (4.12) on the covariant derivatives $\widehat{\nabla}_{\alpha}$, the Bianchi identities in this theory may lead to further constraints on some fields 5 . For theories described by covariant derivatives $\widehat{\nabla}_{\alpha}$, the Bianchi identities are simply Jacobi identities:

$$
\left[\widehat{\nabla}_{[\alpha},\left[\widehat{\nabla}_{\beta}, \widehat{\nabla}_{\gamma)}\right\}\right\}=0
$$

where [, $\}$ is the graded commutator, and [, ) stands for the graded antisymmetrization symbol. Instead of deriving derivative constraints on the spinor fields $\lambda_{\alpha}$ and $\widetilde{\lambda}_{\alpha}$ directly from the Jacobi identities (4.13) we use an equivalent approach which is much easier. While substituting (4.11) into (4.8) one also finds terms proportional to gauge symmetry generators $\mathcal{Y}$ and $\mathcal{Y}^{\prime}$. Vanishing of these terms leads to a set of constraints which is equivalent to the set of constraints obtained from the Jacobi identities (4.13). We outline the result in appendix B.

5 In ordinary field theories, the fields satisfy Bianchi identities because they are expressed in terms of the potentials; they are identities and impose no extra constraints. 
There are two simple solutions to the Jacobi identities corresponding to either $\eta_{\alpha}$ or $\widetilde{\eta}_{\alpha}$ put to zero. An advantage of the first solution is that the covariant derivative $\widehat{\nabla}_{\alpha}$ anti-commutes with itself, $\widehat{\nabla}_{\alpha}^{2}=0$, like in the usual gauged supergravity theories [22]. On the other hand, in the second case we find especially simple form of the anti-commutators $\left\{\widehat{\nabla}_{+}, \widehat{\nabla}_{-}\right\}$and $\left\{\widehat{\nabla}_{+}, \widehat{\nabla}_{\dot{ }}\right\}$. In both cases the remaining spinor superfields can be expressed in terms of an unconstraint real superfield $V$ :

$$
V=\bar{V}
$$

so that the Jacobi identities (4.13) are satisfied. This means that (4.13) impose no further constraints on $V$, and only define the other superfields (like $F$ and $R$ ) in terms of the derivatives of $V$. It is natural to identify the dilaton field with the leading scalar component of $V$ :

$$
\varphi=V \mid
$$

Below we present more evidence for this identification. One might notice that a real superfield $V$ contains one massless vector field, in agreement with the result of the previous section ${ }^{6}$. It is also worthwhile to stress here that massless superfield $V$ is not a Goldstone multiplet itself, but rather what remains after the Goldstone mechanism takes place. A nice property of this solution is that $V$ is manifestly mirror-symmetric.

Since local integration measures of the new $\mathcal{N}=(2,2)$ dilaton supergravity can be nicely derived from the corresponding expressions of the $U(1)_{A} \otimes U(1)_{V}$ theory only for the solution corresponding to $\widetilde{\eta}_{\alpha}=0$, in what follows we discuss in detail only this case. Namely, we take the following ansatz for the spinors $\lambda_{\alpha}$ :

$$
\begin{array}{cc}
\lambda_{+}=\widetilde{\lambda}_{+}=i\left(\widehat{\nabla}_{+} V\right) \quad, \quad \lambda_{-}=-\widetilde{\lambda}_{-}=i\left(\widehat{\nabla}_{-} V\right) \\
\lambda_{\dot{+}}=\widetilde{\lambda}_{\dot{+}}=-i\left(\widehat{\nabla}_{\dot{+}} V\right) \quad, \quad \lambda_{\dot{-}}=-\tilde{\lambda}_{\dot{-}}=-i\left(\widehat{\nabla}_{\dot{-}} V\right)
\end{array}
$$

which implies $\eta_{\alpha}=2 i \widehat{\nabla}_{\alpha} V$ and $\widetilde{\eta}_{\alpha}=0$.

Substituting (4.16) into (4.12), we find the following supergravity algebra:

$$
\left\{\widehat{\nabla}_{+}, \widehat{\nabla}_{+}\right\}=-2\left(\widehat{\nabla}_{+} V\right) \widehat{\nabla}_{+} \quad, \quad\left\{\widehat{\nabla}_{-}, \widehat{\nabla}_{-}\right\}=-2\left(\widehat{\nabla}_{-} V\right) \widehat{\nabla}_{-}
$$

6 However, massless vector fields in two dimensions do not have propagating degrees of freedom. For the same reason two-dimensional superfield $V$ does not have an irreducible transverse component, unlike a similar four-dimensional superfield. 


$$
\begin{gathered}
\left\{\widehat{\nabla}_{+}, \widehat{\nabla}_{-}\right\}=-\frac{1}{2} \bar{R} \mathcal{X} \quad, \quad\left\{\widehat{\nabla}_{+}, \widehat{\nabla}_{\dot{-}}\right\}=-\frac{1}{2} \bar{F} \mathcal{X} \\
\left\{\widehat{\nabla}_{+}, \widehat{\nabla}_{\dot{+}}\right\}=i \widehat{\nabla}_{\ddagger}+\left(\widehat{\nabla}_{\dot{+}} V\right) \widehat{\nabla}_{+}+\left(\widehat{\nabla}_{+} V\right) \widehat{\nabla}_{\dot{+}} \\
\left\{\widehat{\nabla}_{-}, \widehat{\nabla}_{\dot{-}}\right\}=i \widehat{\nabla}_{=}+\left(\widehat{\nabla}_{\dot{-}} V\right) \widehat{\nabla}_{-}+\left(\widehat{\nabla}_{-} V\right) \widehat{\nabla}_{\dot{-}}
\end{gathered}
$$

where the superfields $\bar{R}$ and $\bar{F}$ can be obtained from the Bianchi identities. Solving the set of constraints in appendix B we get:

$$
\bar{R}=4 \widehat{\nabla}_{-} \widehat{\nabla}_{+} V \quad, \quad \bar{F}=4 \widehat{\nabla}_{-} \hat{\nabla}_{+} V
$$

We also find the following expressions for the gauge connection:

$$
\begin{gathered}
\lambda_{\ddagger}=\widetilde{\lambda}_{\ddagger}=i \widehat{\nabla}_{\ddagger} V-2 \widehat{\nabla}_{+} \widehat{\nabla}_{\dot{+}} V+4\left(\widehat{\nabla}_{+} V\right)\left(\widehat{\nabla}_{\dot{+}} V\right) \\
\lambda_{=}=-\widetilde{\lambda}_{=}=i \widehat{\nabla}_{=} V-2 \widehat{\nabla}_{-} \widehat{\nabla}_{\dot{-}} V+4\left(\widehat{\nabla}_{-} V\right)\left(\widehat{\nabla}_{\dot{-}} V\right)
\end{gathered}
$$

Further commutators of the covariant derivatives with vector indices follow from the consistency of the Bianchi identities (4.13):

$$
\begin{aligned}
& {\left[\widehat{\nabla}_{+}, \widehat{\nabla}_{\ddagger}\right]=-\left(2 i \lambda_{+} \lambda_{\dot{+}}+\left(\widehat{\nabla}_{\dot{+}} \lambda_{+}\right)+\left(\widehat{\nabla}_{+} \lambda_{\dot{+}}\right)\right) \widehat{\nabla}_{+}} \\
& {\left[\widehat{\nabla}_{\dot{+}}, \widehat{\nabla}_{\ddagger}\right]=\left(2 i \lambda_{+} \lambda_{\dot{+}}+\left(\widehat{\nabla}_{\dot{+}} \lambda_{+}\right)+\left(\widehat{\nabla}_{+} \lambda_{\dot{+}}\right)\right) \widehat{\nabla}_{\dot{+}}} \\
& {\left[\widehat{\nabla}_{-}, \widehat{\nabla}_{=}\right]=-\left(2 i \lambda_{-} \lambda_{\dot{-}}+\left(\widehat{\nabla}_{\dot{-}} \lambda_{-}\right)+\left(\widehat{\nabla}_{-} \lambda_{\dot{-}}\right)\right) \widehat{\nabla}_{-}} \\
& {\left[\widehat{\nabla}_{\dot{-}}, \widehat{\nabla}_{=}\right]=\left(2 i \lambda_{-} \lambda_{\dot{-}}+\left(\widehat{\nabla}_{\dot{-}} \lambda_{-}\right)+\left(\widehat{\nabla}_{-} \lambda_{\dot{-}}\right)\right) \widehat{\nabla}_{\dot{-}}} \\
& {\left[\widehat{\nabla}_{+}, \widehat{\nabla}_{=}\right]=-\left(\frac{i}{2}\left(\widehat{\nabla}_{-} \bar{R}\right)+\frac{i}{2}\left(\widehat{\nabla}_{-} \bar{F}\right)+\frac{1}{2} \lambda_{-} \bar{R}-\frac{1}{2} \lambda_{-} \bar{F}\right) \mathcal{X}-\frac{i}{2} \bar{R} \widehat{\nabla}_{-}-\frac{i}{2} \bar{F} \widehat{\nabla}_{-}} \\
& {\left[\widehat{\nabla}_{\dot{+}}, \widehat{\nabla}_{=}\right]=\left(\frac{i}{2}\left(\widehat{\nabla}_{-} R\right)+\frac{i}{2}\left(\widehat{\nabla}_{-} F\right)-\frac{1}{2} \lambda_{-} R+\frac{1}{2} \lambda_{-} F\right) \mathcal{X}+\frac{i}{2} R \widehat{\nabla}_{-}+\frac{i}{2} F \widehat{\nabla}_{\dot{-}}} \\
& {\left[\widehat{\nabla}_{-}, \widehat{\nabla}_{\ddagger}\right]=-\left(\frac{i}{2}\left(\widehat{\nabla}_{\dot{+}} \bar{R}\right)+\frac{i}{2}\left(\widehat{\nabla}_{+} F\right)+\frac{1}{2} \lambda_{\dot{+}} \bar{R}-\frac{1}{2} \lambda_{+} F\right) \mathcal{X}+\frac{i}{2} \bar{R} \widehat{\nabla}_{\dot{+}}+\frac{i}{2} F \widehat{\nabla}_{+}} \\
& {\left[\widehat{\nabla}_{\dot{-}}, \widehat{\nabla}_{\ddagger}\right]=\left(\frac{i}{2}\left(\widehat{\nabla}_{+} R\right)+\frac{i}{2}\left(\widehat{\nabla}_{\dot{+}} \bar{F}\right)-\frac{1}{2} \lambda_{+} R+\frac{1}{2} \lambda_{\dot{+}} \bar{F}\right) \mathcal{X}-\frac{i}{2} R \widehat{\nabla}_{+}-\frac{i}{2} \bar{F} \widehat{\nabla}_{\dot{+}}}
\end{aligned}
$$

where we used (4.17). It is worthwhile to stress here that one would obtain a different result de-gauging the corresponding commutators in the $U(1)_{A} \otimes U(1)_{V}$ non-minimal supergravity [26]. 


\section{Lagrangians for Matter Multiplets Coupled to $\mathcal{N}=2$ Dilaton Supergravity}

In order to couple matter fields to new $\mathcal{N}=(2,2)$ dilaton supergravity we have to repeat the analysis of [22]. Up to terms with two derivatives or four fermions, the most general action of $\mathcal{N}=(2,2)$ supergravity coupled to chiral superfields $\Phi_{i}$ and $\mathcal{Z}_{k}$, and twisted chiral superfields $\Sigma_{j}$ looks like (3.15):

$$
\begin{gathered}
S=\int d^{2} x \int d^{2} \theta d^{2} \bar{\theta} E^{-1} \mathcal{L}\left(\Phi_{i}, \bar{\Phi}_{\bar{i}}, \Sigma_{j}, \bar{\Sigma}_{j}, \mathcal{Z}_{k}, \overline{\mathcal{Z}}_{\bar{k}}\right)+ \\
+\int d^{2} x \int d^{2} \theta \mathcal{E}^{-1} W\left(\Phi_{i}\right)+\int d^{2} x \int d \theta^{+} d \theta^{-} \widetilde{\mathcal{E}}^{-1} \widetilde{W}\left(\Sigma_{j}\right)+\text { c.c. }
\end{gathered}
$$

In order to obtain the component action corresponding to (5.1), one needs the appropriate projection formulas. For gauged $\mathcal{N}=(2,2)$ supergravity theories such formulas were derived by Grisaru and Wehlau [6]. In the case of the minimal $U(1)_{A}$ theory the local density projection formula has the following form:

$$
\begin{gathered}
\int d^{2} x d^{4} \theta E^{-1} \mathcal{L}=\int d^{2} x e^{-1}\left[\nabla^{2}+i \psi_{=}^{\dot{\Xi}} \nabla_{+}-i \psi_{\ddagger}^{\dot{+}} \nabla_{-}+\right. \\
\left.+\left(-\frac{1}{2} \bar{H}-\psi_{\ddagger}^{\dot{\dot{亠}}} \psi_{=}^{\dot{\dot{ }}}+\psi_{=}^{\dot{\dot{ }}} \psi_{\ddagger}^{\dot{+}}\right)\right] \bar{\nabla}^{2} \mathcal{L} \mid
\end{gathered}
$$

Here $\psi_{\mu}^{\alpha}$ is the gravitino field and $\mathcal{L}$ is an arbitrary scalar function of superfields. In fact, the same projection formula is also valid in the non-minimal $U(1)_{A} \otimes U(1)_{V}$ supergravity theory [22]. Although (5.2) is a D-type superinvariant, sometimes it is called a chiral density projector because in the non-minimal $\mathcal{N}=(2,2)$ supergravity $\bar{\nabla}^{2} \mathcal{L}$ is a chiral

superfield (for a general $\mathcal{L}$ ). Therefore, replacing $\bar{\nabla}^{2} \mathcal{L}$ by an arbitrary covariantly chiral Lagrangian $\mathcal{L}_{c}$, we can obtain the component projection formula for any chiral superspace integral:

$$
\begin{aligned}
& \int d^{2} x d^{2} \theta \mathcal{E}^{-1} \mathcal{L}_{c}=\int d^{2} x e^{-1}\left[\nabla^{2}+i \psi_{=}^{\dot{\doteq}} \nabla_{+}-i \psi_{\ddagger}^{\dot{+}} \nabla_{-}+\right. \\
& \left.+\left(-\frac{1}{2} \bar{H}-\psi_{\ddagger}^{\dot{\bar{\xi}}} \psi_{=}^{\dot{+}}+\psi_{=}^{\dot{\dot{ }}} \psi_{\ddagger}^{\dot{+}}\right)\right] \mathcal{L}_{c} \mid
\end{aligned}
$$

In particular, the superspace measures $E^{-1}$ and $\mathcal{E}^{-1}$ are related as follows:

$$
\int d^{2} x d^{4} \theta E^{-1} \mathcal{L}=\int d^{2} x d^{2} \theta \mathcal{E}^{-1} \bar{\nabla}^{2} \mathcal{L} \mid
$$

By mirror symmetry the twisted chiral density projection formula in the $U(1)_{V}$ gauged supergravity theory has the following form:

$$
\int d^{2} x d^{4} \theta E^{-1} \mathcal{L}=\int d^{2} x e^{-1}\left[\nabla_{\dot{-}} \nabla_{+}-i \psi_{=}^{-} \nabla_{+}+i \psi_{\ddagger}^{\dot{+}} \nabla_{\dot{-}}+\right.
$$




$$
\left.+\left(-\frac{1}{2} \bar{G}+\psi_{\ddagger}^{-} \psi_{=}^{\dot{+}}+\psi_{\ddagger}^{\dot{+}} \psi_{=}^{-}\right)\right] \nabla_{\dot{+}} \nabla_{-} \mathcal{L} \mid
$$

As explained in [22], the derivation of this formula goes through as in [6] for the case of $U(1)_{A}$ theory. In the case of the $U(1)_{V} \otimes U(1)_{A}$ gauged supergravity the symmetry between chiral and twisted chiral fields is restored by the contribution of the anticommutator term $\left\{\nabla_{+}, \nabla_{\dot{-}}\right\} \sim \bar{F} \mid$. More explicitly, the twisted chiral density projection formula (5.5) can be derived using the methods of [6] or [27].

The projection formulas in the two-dimensional $\mathcal{N}=(2,2)$ dilaton supergravity can be obtained from (5.2) and (5.5) replacing $\nabla_{\alpha}$ by a new covariant derivative $\widehat{\nabla}_{\alpha}$. Thus, substituting (4.11) and (4.16) into (5.2) and using the commutation relations (4.7) - (4.8) we obtain the following density projection formula:

$$
\begin{aligned}
& \int d^{2} x d^{4} \theta E^{-1} \mathcal{L}=\int d^{2} x e^{-1}\left[\left(\widehat{\nabla}_{+}-\left(\widehat{\nabla}_{+} V\right)\right)\left(\widehat{\nabla}_{-}-\left(\widehat{\nabla}_{-} V\right)\right)+i \psi \dot{\dot{=}}\left(\widehat{\nabla}_{+}-\left(\widehat{\nabla}_{+} V\right)\right)-\right. \\
& \left.-i \psi_{\ddagger}^{\dot{+}}\left(\widehat{\nabla}_{-}-\left(\widehat{\nabla}_{-} V\right)\right)+\left(-\frac{1}{2} \bar{H}-\psi_{\ddagger}^{\dot{\dot{ }}} \psi_{=}^{\dot{+}}+\psi_{=}^{\dot{\dot{\dot{ }}}} \psi_{\neq}^{\dot{+}}\right)\right] \widehat{\bar{\nabla}}^{2} \mathcal{L} \mid= \\
& =\int d^{2} x e^{-1}\left[\widehat{\nabla}_{+} \widehat{\nabla}_{-}+i\left(\psi_{=}^{\dot{\dot{ }}}-\lambda_{-}\right) \widehat{\nabla}_{+}-i\left(\psi_{\neq}^{\dot{+}}-\lambda_{+}\right) \widehat{\nabla}_{-}+\right. \\
& \left.+\left(-\frac{1}{4} \bar{H}-\psi_{\neq}^{\dot{\bar{q}}} \psi_{=}^{\dot{+}}+\left(\psi_{=}^{\dot{\dot{ }}}-\lambda_{-}\right)\left(\psi_{\neq}^{\dot{+}}-\lambda_{+}\right)\right)\right] \widehat{\nabla}_{\dot{+}} \widehat{\nabla}_{\dot{-}} \mathcal{L} \mid
\end{aligned}
$$

In order to convince even hard boiled sceptics that (5.6) is the right projector, in appendix D we repeat the calculation of Grisaru and Wehlau [6] in the new $\mathcal{N}=(2,2)$ dilaton supergravity. As expected, the result is equivalent to (5.6).

Similarly, the twisted chiral density projection formula (5.5) yields:

$$
\begin{gathered}
\int d^{2} x d^{4} \theta E^{-1} \mathcal{L}=\int d^{2} x e^{-1}\left[\left(\widehat{\nabla}_{\dot{-}}-\left(\widehat{\nabla}_{\dot{-}} V\right)\right)\left(\widehat{\nabla}_{+}-\left(\widehat{\nabla}_{+} V\right)\right)-i \psi_{=}^{-}\left(\widehat{\nabla}_{+}-\left(\widehat{\nabla}_{+} V\right)\right)+\right. \\
\left.+i \psi_{\mp}^{\dot{+}}\left(\widehat{\nabla}_{\dot{-}}-\left(\widehat{\nabla}_{\dot{-}} V\right)\right)+\left(-\frac{1}{2} \bar{G}+\psi_{\mp}^{-} \psi_{=}^{\dot{+}}+\psi_{\ddagger}^{\dot{+}} \psi_{=}^{-}\right)\right] \widehat{\nabla}_{\dot{+}} \widehat{\nabla}_{-} \mathcal{L} \mid
\end{gathered}
$$

We note that for a given superspace Lagrangian $\mathcal{L}$, both projection formulas (5.6) and (5.7) lead to the same result:

$$
\begin{gathered}
\int d^{2} x d^{4} \theta E^{-1} \mathcal{L}=\int d^{2} x e^{-1}\left[\left(\widehat{\nabla}_{+}-\left(\widehat{\nabla}_{+} V\right)\right)\left(\widehat{\nabla}_{-}-\left(\widehat{\nabla}_{-} V\right)\right)+i \psi \stackrel{\dot{\doteq}}{=}\left(\widehat{\nabla}_{+}-\left(\widehat{\nabla}_{+} V\right)\right)-\right. \\
\left.-i \psi_{\ddagger}^{\dot{+}}\left(\widehat{\nabla}_{-}-\left(\widehat{\nabla}_{-} V\right)\right)+\left(-\frac{1}{2} \bar{H}-\psi_{\ddagger}^{\dot{\dot{y}}} \psi_{=}^{\dot{\dot{+}}}+\psi_{=}^{\dot{\dot{j}}} \psi_{\ddagger}^{\dot{+}}\right)\right] \widehat{\bar{\nabla}}^{2} \mathcal{L} \mid=
\end{gathered}
$$




$$
\begin{aligned}
=\int & d^{2} x e^{-1}\left[\left(\widehat{\nabla}_{\dot{-}}-\left(\widehat{\nabla}_{\dot{-}} V\right)\right)\left(\widehat{\nabla}_{+}-\left(\widehat{\nabla}_{+} V\right)\right)-i \psi_{=}^{-}\left(\widehat{\nabla}_{+}-\left(\widehat{\nabla}_{+} V\right)\right)+\right. \\
& \left.+i \psi_{\neq}^{\dot{+}}\left(\widehat{\nabla}_{\dot{-}}-\left(\widehat{\nabla}_{\dot{-}} V\right)\right)+\left(-\frac{1}{2} \bar{G}+\psi_{\mp}^{-} \psi_{=}^{\dot{+}}+\psi_{\mp}^{\dot{+}} \psi_{=}^{-}\right)\right] \widehat{\nabla}_{\dot{+}} \widehat{\nabla}_{-} \mathcal{L} \mid
\end{aligned}
$$

This follows from the corresponding property of the local density projectors in gauged $\mathcal{N}=$ $(2,2)$ supergravity theory [6], and also can be verified explicitly using the commutation relations (4.17) in the new $\mathcal{N}=(2,2)$ dilaton supergravity.

Now we are ready to derive component actions for various superspace Lagrangians $\mathcal{L}$. Let us start with a simple example corresponding to pure dilaton supergravity. Obviously, in order to reproduce the right exponential dependence on the dilaton field in (3.5), we have to take the function $\mathcal{L}$ in the form:

$$
\mathcal{L}_{\text {grav }}=\exp (-2 V)
$$

Substituting this in the projection formula (5.6) (or (5.7)) and seting all the fermions to zero, we obtain the following action for bosonic fields:

$$
S_{\text {grav }}=\int d^{2} x e^{-1} \exp (-2 \varphi)\left[R^{(2)}+4\left(\partial_{\mu} \varphi\right)\left(\partial^{\mu} \varphi\right)\right]
$$

Here we used (4.16) and the formulas for the other components of the real superfield $V$ derived in appendix C. Clearly, the action (5.8) for dilaton and graviton fields agreest with the first two terms in the effective action (3.5) of Type IIA theory on a Calabi-Yau four-fold. Moreover, we note that bosonic action (5.8) has exactly the same form as the action of $\mathcal{N}=0$ dilaton gravity studied long time ago, see e.g. [24].

Now we consider coupling of $\mathcal{N}=(2,2)$ dilaton supergravity to matter fields. In particular, we are interested in superspace form of the effective action (3.5) describing compactification of Type IIA string theory on a Calabi-Yau four-fold $X$. Once again, to reproduce the exponential dependence on $\varphi=V \mid$ we take the superspace action in the form:

$$
\int d^{2} x d^{4} \theta E^{-1} \exp (-2 V) \mathcal{L}
$$

where $\mathcal{L}$ is a function of all matter superfields but $V$. It is convenient to absorb $\exp (-2 V)$ in the definition of the supervielbein determinant $E_{0}^{-1}=E^{-1} \exp (-2 V)$, so that $\mathcal{L}=1$

7 We will account for the extra volume factor $\mathcal{V}$ in a moment. 
corresponds to pure supergravity action (5.8), like in $\mathcal{N}=1$ four-dimensional theory. Commuting $\exp (-2 V)$ to the left in (5.6), we find the modified projection formula:

$$
\begin{gathered}
\int d^{2} x d^{4} \theta E_{0}^{-1} \mathcal{L}= \\
=\int d^{2} x e^{-1} \exp (-2 \varphi)\left[\left(\widehat{\nabla}_{+}-3\left(\widehat{\nabla}_{+} V\right)\right)\left(\widehat{\nabla}_{-}-3\left(\widehat{\nabla}_{-} V\right)\right)+i \psi_{=}^{\dot{\Xi}}\left(\widehat{\nabla}_{+}-3\left(\widehat{\nabla}_{+} V\right)\right)-\right. \\
\left.-i \psi_{\ddagger}^{\dot{+}}\left(\widehat{\nabla}_{-}-3\left(\widehat{\nabla}_{-} V\right)\right)+\left(-\frac{1}{2} \bar{H}-\psi_{\ddagger}^{\dot{-}} \psi_{=}^{\dot{+}}+\psi_{=}^{\dot{\dot{\Xi}}} \psi_{\ddagger}^{\dot{1}}\right)\right]\left(\widehat{\nabla}_{\dot{+}}-2\left(\widehat{\nabla}_{\dot{+}} V\right)\right)\left(\widehat{\nabla}_{\dot{-}}-2\left(\widehat{\nabla}_{\dot{-}} V\right)\right) \mathcal{L} \mid
\end{gathered}
$$

Applying this projection formula to an arbitrary function $\mathcal{L}\left(\Phi_{i}, \bar{\Phi}_{\bar{i}}, \Sigma_{j}, \bar{\Sigma}_{j}\right)$ of chiral superfields $\Phi_{i}$ and twisted chiral superfields $\Sigma_{j}$ we get the action of the bosonic fields (the vielbein determinant $e^{-1}$ is suppressed):

$$
\begin{gathered}
L=e^{-2 \varphi} \mathcal{L}\left[R^{(2)}+4 \partial_{\mu}\left(\varphi-\frac{1}{2} \log \mathcal{L}\right) \partial^{\mu}\left(\varphi-\frac{1}{2} \log \mathcal{L}\right)+\right. \\
+\frac{1}{2}(\log \mathcal{L})_{\phi_{i} \bar{\phi}_{j}}\left(\partial_{\mu} \phi_{i}\right)\left(\partial^{\mu} \bar{\phi}_{j}\right)-\frac{1}{2}(\log \mathcal{L})_{\sigma_{i} \bar{\sigma}_{j}}\left(\partial_{\mu} \sigma_{i}\right)\left(\partial^{\mu} \bar{\sigma}_{j}\right)+ \\
\left.+\frac{1}{2} \epsilon^{\mu \nu}\left((\log \mathcal{L})_{\phi_{i} \bar{\sigma}_{j}}\left(\partial_{\mu} \phi_{i}\right)\left(\partial_{\nu} \bar{\sigma}_{j}\right)+(\log \mathcal{L})_{\sigma_{j} \bar{\phi}_{i}}\left(\partial_{\mu} \bar{\phi}_{i}\right)\left(\partial_{\nu} \sigma_{j}\right)\right)\right]
\end{gathered}
$$

The subscripts on $\mathcal{L}$ denote derivatives with respect to the scalar fields, e.g. $(\log \mathcal{L})_{\phi_{i} \bar{\phi}_{j}}=$ $\left(\partial^{2} / \partial \phi_{i} \partial \bar{\phi}_{j}\right) \log \mathcal{L}$. Deriving (5.11) one may find helpful some formulas from appendix D where we discuss in detail the component action of a free chiral superfield. A careful reader may notice that (5.11) has the structure reminiscent of $\mathcal{N}=1$ supergravity in four dimensions. In particular, it is convenient to introduce the Kähler potential $K$ :

$$
\mathcal{L}=\exp (-K)
$$

so that the superspace action (5.9) takes the form:

$$
\int d^{2} x d^{4} \theta E_{0}^{-1} e^{-K}=\int d^{2} x d^{4} \theta E^{-1} e^{-2 V} e^{-K} .
$$

Performing the superspace integration, one finds Lagrangian for the bosonic fields:

$$
\begin{gathered}
L=e^{-2 \widetilde{\varphi}}\left[R^{(2)}+4\left(\partial_{\mu} \widetilde{\varphi}\right)\left(\partial^{\mu} \widetilde{\varphi}\right)-\frac{1}{2} K_{\phi_{i} \bar{\phi}_{j}}\left(\partial_{\mu} \phi_{i}\right)\left(\partial^{\mu} \bar{\phi}_{j}\right)+\right. \\
\left.+\frac{1}{2} K_{\sigma_{i} \bar{\sigma}_{j}}\left(\partial_{\mu} \sigma_{i}\right)\left(\partial^{\mu} \bar{\sigma}_{j}\right)-\frac{1}{2} \epsilon^{\mu \nu}\left(K_{\phi_{i} \bar{\sigma}_{j}}\left(\partial_{\mu} \phi_{i}\right)\left(\partial_{\nu} \bar{\sigma}_{j}\right)+K_{\sigma_{j} \bar{\phi}_{i}}\left(\partial_{\mu} \bar{\phi}_{i}\right)\left(\partial_{\nu} \sigma_{j}\right)\right)\right]
\end{gathered}
$$


where we introduced a new dilaton field $\widetilde{\varphi}=\varphi+\frac{1}{2} K$ invariant under generalized Kähler transformations 3.7$)$ :

$$
K \longrightarrow K+\Lambda_{1}\left(\phi_{i}, \sigma_{j}\right)+\bar{\Lambda}_{1}\left(\bar{\phi}_{\bar{i}}, \bar{\sigma}_{j}\right)+\Lambda_{2}\left(\phi_{i}, \bar{\sigma}_{j}\right)+\bar{\Lambda}_{2}\left(\bar{\phi}_{\bar{i}}, \sigma_{j}\right)
$$

Under this Kähler transformation the original dilaton field $\varphi$ is shifted in the opposite way, so that the linear combination $\widetilde{\varphi}=\varphi+\frac{1}{2} K$ remains invariant. Since the Kähler metric is invariant under (5.15) as well, both the superspace action (5.13) and the corresponding component action (5.14) are manifestly invariant under the generalized Kähler transformations (5.15).

Now we are in position to identify the function $K$ that would reproduce the effective action (3.5) of Type IIA theory on a Calabi-Yau four-fold. Namely, the superspace action (5.13) gives the effective action (3.5) if $K$ is the total Kähler potential (3.9). This form of the superspace action might be expected for a number of reasons. First of all, it is similar to the superspace action of $\mathcal{N}=1$ supergravity in four dimensions. Moreover, Type IIA supergravity on a Calabi-Yau four-fold has a breathing mode corresponding to rescaling of the volume $\mathcal{V} \rightarrow c^{2} \mathcal{V}$ and simultaneous shift of the dilaton $\varphi \rightarrow \varphi+\log c, c f$. (3.1). Therefore, the superspace action is expected to be a function of $2 V+K$, where $K$ is the total Kähler potential given by $(3.9)$ - (3.10).

To summarize, we constructed superspace Lagrangians describing $\mathcal{N}=(2,2)$ dilaton supergravity coupled to matter superfields and found projection formulas that allow one to rewrite integrals over the entire superspace in terms of component fields. Therefore, we provide a superspace formulation of the effective field theories constructed from compactification of Type IIA string theory on Calabi-Yau four-folds, as well as more general $\mathcal{N}=(2,2)$ sigma-models with torsion coupled to dilaton supergravity, $c f$. [5]].

Incorporation of superpotential terms is more subtle. These terms are superinvariants obtained by integration only over a half of the superspace, $c f$. (5.1). Unfortunately, unlike (5.2) and (5.5), the chiral and twisted chiral density projectors in $\mathcal{N}=(2,2)$ dilaton supergravity do not simply follow from the full superspace projector (5.10). However, by dimensional arguments and from an examination of the index structure of the possible terms, the chiral density projection formula must look like:

$$
\int d^{2} x \int d^{2} \theta \mathcal{E}^{-1} W=\int d^{2} x e^{-1}\left[\widehat{\nabla}^{2}+\ldots-\frac{1}{2} \bar{H}-\psi_{\ddagger}^{\dot{-}} \psi_{=}^{\dot{+}}+\psi_{=}^{\dot{\dot{ }}} \psi_{\ddagger}^{\dot{+}}\right] W \mid
$$


where the dots stand for term containing $\lambda_{\alpha}$ or terms linear in covariant derivatives 8 . By the similar reasoning, the twsited chiral density projection formula must look like (5.5):

$$
\int d^{2} x \int d^{2} \theta \widetilde{\mathcal{E}}^{-1} \widetilde{W}=\int d^{2} x e^{-1}\left[\widehat{\nabla}_{\dot{-}} \widehat{\nabla}_{+}+\ldots-\frac{1}{2} \bar{G}+\psi_{\ddagger}^{-} \psi_{=}^{\dot{+}}+\psi_{\ddagger}^{\dot{+}} \psi_{=}^{-}\right] \widetilde{W} \mid
$$

Although normalization and coefficients in (5.16) and (5.17) may not be correct, the terms quadratic in the gravitino are rather general and, in particular, are independent on degauging. So, we infer that one effect of the superpotential is to produce a mass term for the gravitino fields [22]:

$$
m_{\psi_{\ddagger} \dot{\bar{\prime}}} \sim W, \quad m_{\psi_{\ddagger}^{-}} \sim \widetilde{W}
$$

It is this property of $\mathcal{N}=(2,2)$ supergravity that was needed in [13, 14 in order to find the superpotentials (3.13) and (3.14) induced by Ramond-Ramond fluxes. Moreover, let us demonstrate that (5.16) and (5.17) lead to the expected structure of the scalar potential (3.18). Extending the computation of (5.11), from (5.1) we get the action of the auxiliary fields:

$$
\begin{gathered}
L_{\mathrm{aux}} \sim \mathcal{L}\left[\left|\frac{1}{2} H-i \bar{A}_{i}(\log \mathcal{L})_{\phi_{i}}\right|^{2}+\left|\frac{1}{2} G-i \bar{B}_{j}(\log \mathcal{L})_{\sigma_{j}}\right|^{2}+(\log \mathcal{L})_{\phi_{i} \bar{\phi}_{j}} A_{i} \bar{A}_{j}-\right. \\
\left.-(\log \mathcal{L})_{\sigma_{i} \bar{\sigma}_{j}} B_{i} \bar{B}_{j}\right]-i\left(W_{\phi_{i}}-(\log \mathcal{L})_{\phi_{i}}\right) A_{i}-W\left(\frac{1}{2} \bar{H}+i A_{i}(\log \mathcal{L})_{\phi_{i}}\right)- \\
-i\left(\widetilde{W}_{\sigma_{i}}-(\log \mathcal{L})_{\sigma_{i}}\right) B_{i}-\widetilde{W}\left(\frac{1}{2} \bar{G}+i B_{i}(\log \mathcal{L})_{\sigma_{i}}\right)+\text { c.c. }
\end{gathered}
$$

Integrating out the auxiliary fields and using (5.12), we find the expected scalar potential (3.18):

$$
L_{\mathrm{aux}} \sim e^{K}\left(K_{\phi_{i} \bar{\phi}_{j}}^{-1}\left(D_{\phi_{i}} W\right)\left(D_{\bar{\phi}_{j}} \bar{W}\right)-K_{\sigma_{i} \bar{\sigma}_{j}}^{-1}\left(D_{\sigma_{i}} \widetilde{W}\right)\left(D_{\bar{\sigma}_{j}} \overline{\widetilde{W}}\right)-|W|^{2}-|\widetilde{W}|^{2}\right)
$$

with the covariant derivatives 3.17$)$.

\section{Compactification of Type IIB String Theory on Calabi-Yau Four-folds}

Compactification of Type IIB string theory on a Calabi-Yau four-fold $X$ leads to a chiral $\mathcal{N}=(0,4)$ supersymmetric effective field theory in two non-compact dimensions. In the low-energy limit this theory is described by $\mathcal{N}=(0,4)$ supergravity coupled to scalar

superfields. In this section we perform a Kaluza-Klein reduction on a Calabi-Yau four-fold

8 These terms will not affect the action of bosonic fields. 
$X$ and, in particular, find that the supergravity multiplet includes a real scalar dilaton field instead of an $S U(2)$ gauge field [28,29]. A manifestly supersymmetric formulation of such $\mathcal{N}=(0,4)$ supergravity theory will be presented in the next section. In this section we show that Kaluza-Klein harmonics combine into $\mathcal{N}=(0,4)$ scalar superfields [29, 30]. Furthermore, since we deal with $\mathcal{N}=(0,4)$ supersymmetry, there is a significant difference between right-movers and left-movers. Namely, all left-moving modes are supersymmetry singlets. We will also see that the difference between the zero-point energy of the leftmovers and the right-movers is proportional to the Euler number of $X$ [31].

In the large volume limit the bosonic spectrum of light modes in Type IIB string theory includes the metric $g_{M N}$, the dilaton $\varphi$, the axion $l$, the 4 -form tensor $D_{M N P Q}$ and two tensor fields $B_{M N}^{R R}$ and $B_{M N}^{N S}$. Together with the fermionic superpartners all these fields fit into Type IIB supergravity multiplet. Non-perturbative Type IIB string theory is invariant under $S L(2, \mathbb{Z})$ duality group. In order to see the action of this group on the supergravity fields, it is convenient to define the following quantities:

$$
\begin{gathered}
\lambda=l+i e^{-\varphi} \\
\mathbf{M}=\frac{1}{\operatorname{Im} \lambda}\left(\begin{array}{cc}
|\lambda|^{2} & -\operatorname{Re} \lambda \\
-\operatorname{Re} \lambda & 1
\end{array}\right) \\
\mathbf{H}_{M N P}=\left(\begin{array}{c}
\partial_{[M} B_{N P]}^{N S} \\
\partial_{[M} B_{N P]}^{R R}
\end{array}\right)
\end{gathered}
$$

and

$$
F_{M N P Q R}=\partial_{[M} D_{N P Q R]}+\frac{3}{4} B_{[M N}^{N S} \partial_{P} B_{Q R]}^{R R}
$$

Then, the field strength $F_{M N P Q R}$ is a singlet under the $S L(2, \mathbb{Z})$ duality group, while $\mathbf{H}$ transforms as a "vector". Finally, $S L(2, \mathbb{Z})$ acts on a complex scalar $\lambda$ in the usual way:

$$
\lambda \rightarrow \frac{a \lambda+b}{c \lambda+d}
$$

where the integer numbers $a, b, c$ and $d$ satisfy $a d-b c=1$.

The five-form field strength $F$ is self-dual:

$$
F=* F
$$

Although this equation can not be derived from any action, for a moment we ignore this subtlety and write bosonic "Type IIB supergravity Lagrangian" simply as:

$$
L_{(10)}=\sqrt{-g}\left[-\frac{1}{4} R^{(10)}+\frac{1}{16} \operatorname{Tr}(\partial \mathbf{M} \cdot \partial \mathbf{M})+\frac{3}{16} \mathbf{H}^{T} \cdot \mathbf{M} \cdot \mathbf{H}+\frac{5}{24} F^{2}+\ldots\right]
$$


where the dots stand for higher derivative terms.

As in Type IIA theory, in order to find the zero-mode spectrum we have to expand Type IIB supergravity fields in harmonic $(p, q)$-forms on the space $X$. The metric modes are exactly the same as in (2.3). Namely, from the reduction of $g_{M N}$ we find $h^{1,1}$ real scalars $s_{i}, h^{3,1}$ complex scalars $\phi_{j}$ and the two-dimensional metric $g_{\mu \nu}$. It turns out that one of the scalars $s_{i}$ comes into the two-dimensional supergravity multiplet. Namely, it is the Kaluza-Klein mode corresponding to the volume of the Calabi-Yau four-fold:

$$
\mathcal{V}=d_{i j k l} s^{i} s^{j} s^{k} s^{l}
$$

where $d_{i j k l}$ are the intersection numbers of $X$ given by (2.7). With this mode excluded, the Kähler deformations of the metric yield $h^{1,1}-1$ scalars $\check{s}_{i}=\mathcal{V}^{-\frac{1}{4}} s_{i}$ satisfying the condition:

$$
d_{i j k l} \check{s}^{i} \check{s}^{j} \check{s}^{k} \check{s}^{l}=1
$$

Expanding the doublet of tensor fields as:

$$
B^{N S}=\sum_{i=1}^{h^{1,1}} r_{i} \omega_{i}^{(1,1)}, \quad B^{R R}=\sum_{i=1}^{h^{1,1}} t_{i} \omega_{i}^{(1,1)}
$$

we get pairs of real scalars $r_{i}$ and $t_{i}, h^{1,1}$ in number. All these modes are both right-moving and left-moving.

Expansion of the self-dual field $D$ is a bit subtle. Namely, instead of $D$ one has to expand the field strength $F \sim \sum_{i} \partial_{\mu} u_{i} \cdot \omega_{i}^{(4)}$ and impose the self-duality condition (6.6). Depending on whether the form $\omega_{i}^{(4)} \in H^{4}(X, \mathbb{R})$ is self-dual or anti-self-dual the scalar field $u_{i}$ is left-moving or right-moving, respectively. Therefore, we have to distinguish carefully self-dual and anti-self-dual harmonics of $F$. To this end we recall some topological properties of Calabi-Yau four-folds. There is a decomposition of the space of the middle dimensional forms on $X$ :

$$
H^{4}(X, \mathbb{R})=B_{+}(X) \oplus B_{-}(X)
$$

where we denote by $B_{+}(X)$ (resp. $\left.B_{-}(X)\right)$ the space of (anti-)self-dual 4 -forms on $X$. Let us call the corresponding dimensions $b_{ \pm}=\operatorname{dim} B_{ \pm}(X)$. Then $b_{+}$and $b_{-}$are related by the 
Hirzebruch signature 9 (see e.g. [7]):

$$
\tau(Q)=b_{+}-b_{-}=\frac{1}{45} \int_{X}\left(7 p_{2}-p_{1}^{2}\right)=\frac{\chi}{3}+32
$$

of the quadratic form $Q\left(\omega_{1}, \omega_{2}\right)=\int_{X} \omega_{1} \wedge \omega_{2}$. On the other hand, we also have $b_{4}=$ $b_{+}+b_{-}=2+2 h^{3,1}+h^{2,2}$. Hence, using (2.1) and (2.2) we find:

$$
b_{+}=47+3 h^{1,1}+4 h^{3,1}-2 h^{2,1}
$$

and

$$
b_{-}=-1+h^{1,1}+2 h^{3,1}
$$

Actually, we can be a little bit more precise. All the forms of Hodge type $(3,1)$ or $(1,3)$ are anti-self-dual, while the $(4,0)$ - and $(0,4)$-forms on a Calabi-Yau four-fold are self-dual [13]. Therefore, from (6.9) and (6.10) we find that:

$$
b_{+}^{(2,2)}=45+3 h^{1,1}+4 h^{3,1}-2 h^{2,1}
$$

and

$$
b_{-}^{(2,2)}=h^{1,1}-1
$$

Now we expand the self-dual field strength $F$ as:

$$
F=\sum_{i=1}^{b_{+}^{2,2}}\left(\partial_{\mu} u_{i}\right) \omega_{i}^{(+)}+\sum_{j=1}^{b_{-}^{2,2}}\left(\partial_{\mu} v_{j}\right) \omega_{j}^{(-)}+\sum_{k=1}^{h^{3,1}}\left(\partial_{\mu} p_{k}\right) \omega_{k}^{(3,1)}+\left(\partial_{\mu} q\right) \Omega+\text { c.c. }
$$

where the scalar fields $u_{i}$ and $v_{j}$ are real, while $p_{k}$ and $q$ are complex. As we explained above, $u_{i}$ and $q$ must be left-moving, $v_{j}$ and $p_{k}$ must be right-moving. In particular, the former are singlets with respect to four left supercharges satisfying:

$$
\left\{Q_{+}^{i}, Q_{+}^{j}\right\}=\delta^{i j} P_{+}
$$

9 The explicit form of the Pontryagin classes is given by:

$$
p_{1}=-\frac{1}{2} \operatorname{tr} R^{2}, \quad p_{2}=-\frac{1}{4} \operatorname{tr} R^{4}+\frac{1}{8}\left(\operatorname{tr} R^{2}\right)^{2}
$$


Note that the Kaluza-Klein modes of the self-dual field $F$ associated with $(2,1)$-harmonic forms give 2-form field strengths of massless vector fields in two dimensions and, therefore, do not lead to new propagating degrees of freedom.

Unlike $\mathcal{N}=(2,2)$ theory constructed from compactification of Type IIA string theory, in Type IIB compactification on on a Calabi-Yau four-fold $X$ not all the fermionic modes can be determined by $\mathcal{N}=(0,4)$ supersymmetry. In the right sector we still can use supersymmetry arguments to conclude that two-dimensional supergravity multiplet contains 2ind $(\not D)$ fermions and 2ind $(\not D)$ Rarita-Schwinger fields that come from the corresponding spin- $\frac{1}{2}$ and spin- $\frac{3}{2}$ fields in Type IIB supergravity. On a Calabi-Yau four-fold the Dirac index is given by:

$$
\operatorname{ind}(\not D)=\frac{1}{1440} \int_{X}\left(\frac{7}{4} p_{1}^{2}-p_{2}\right)=2
$$

in accordance with $\mathcal{N}=(0,4)$ supersymmetry. Furthermore, all the right-moving scalars found above $\left(\phi_{i}, \bar{\phi}_{\bar{i}}, p_{i}, \bar{p}_{\bar{i}}, \check{s}_{j}, r_{j}, t_{j}, v_{j}, \varphi\right.$ and $\left.l\right)$ are accompanied by right-moving fermions. Simple counting gives:

$$
n_{+}=4 h^{3,1}+4 h^{1,1}
$$

for the total number of the right-moving fermions 10 . There are also left-moving fermions which are supersymmetry singlets. The number of left-moving fermions, however, is not determined by supersymmetry. So, it has to be computed separately. Since the fermions in question come from the Type IIB gravitinos, their number (minus the number of rightmoving fermions) is given by the Rarita-Schwinger index:

$$
n_{-}-n_{+}=2 \operatorname{ind}\left(\not D_{3 / 2}\right)
$$

Using (6.15) and the explicit expression for the Rarita-Schwinger index on a Calabi-Yau four-fold $X$ :

$$
\operatorname{ind}\left(D_{3 / 2}\right)=\frac{1}{180} \int_{X}\left(\frac{37}{4} p_{1}^{2}-31 p_{2}\right)=-4 h^{1,1}-4 h^{3,1}+4 h^{2,1}
$$

we obtain:

$$
n_{-}=4 h^{2,1}
$$

10 Note that $n_{+}$is divisible by 4 . 
Now we are ready to assemble the supermultiplets. Combining the left-moving bosonic modes with the fermion fields we get the following supermultiplets:

$$
\begin{aligned}
& \text { a gravitational multiplet: } g_{\mu \nu}, \quad \mathcal{V} \\
& h^{3,1} \text { scalar multiplets } \Phi_{i}: \quad \phi_{i}, \quad \bar{\phi}_{i}, \quad p_{i}, \quad \bar{p}_{i} \\
& h^{1,1} \text { scalar multiplets } \Sigma_{j}: \quad \check{s}_{j}, \quad r_{j}, \quad t_{j}, \quad v_{j}, \quad \varphi, \quad l
\end{aligned}
$$

Performing a reduction of the ten-dimensional supersymmetry conditions one can easily check that these fields indeed represent bosonic components of the supermultiplets as stated. Note, all the matter multiplets include four real scalar fields in accordance with the general classification of scalar superfields [28, 32, 29, 30]. However, the content of the gravitational multiplet is different from what was usually studied in $\mathcal{N}=(0,4)$ supergravity theories [28,29]. In compactification of Type IIB string theory on a Calabi-Yau four-fold we find that the gravitational multiplet includes a real scalar $\mathcal{V}$ instead of $S U(2)$ gauge field. In the next section we describe the superspace formulation of this $\mathcal{N}=(0,4)$ supergravity using the Goldstone approach.

In order to find the low-energy effective action one has to substitute (2.3), (6.8) and (6.13) into (6.7). Integrating over the internal space by means of the formulas (2.4), (2.5) we get the following two-dimensional action for bosonic fields:

$$
\begin{gathered}
L_{(2)}=\sqrt{-g} \mathcal{V}\left[-\frac{1}{4} R^{(2)}+\frac{1}{2}\left(\partial_{+} \lambda\right)\left(\partial_{-} \lambda\right)+\frac{1}{2} \mathcal{G}_{\phi_{i} \bar{\phi}_{\bar{j}}}\left(\partial_{+} \phi^{i}\right)\left(\partial_{-} \bar{\phi}^{j}\right)+\frac{1}{2} \mathcal{G}_{\phi_{i} \bar{\phi}_{\bar{j}}}\left(\partial_{0} p^{i}\right)\left(\partial_{+} \bar{p}^{j}\right)+\right. \\
+\frac{1}{2} \mathcal{G}_{\sigma_{i} \bar{\sigma}_{j}}\left(\left(\partial_{+} s^{i}\right)\left(\partial_{-} s^{j}\right)+\left(\partial_{+} r^{i}\right)\left(\partial_{-} r^{j}\right)+\left(\partial_{+} t^{i}\right)\left(\partial_{-} t^{j}\right)+\left(\partial_{0} v^{i}\right)\left(\partial_{+} v^{j}\right)\right)+ \\
\left.+\frac{1}{2} Q_{i j}\left(\partial_{0} u^{i}\right)\left(\partial_{-} u^{j}\right)+\frac{1}{2}\left(\partial_{0} q\right)\left(\partial_{-} q\right)+\text { c.c. }\right]
\end{gathered}
$$

This Lagrangian describes non-linear sigma-model interacting with $\mathcal{N}=(0,4)$ supergravity. The target space of the left-moving fields is the cotangent bundle to the moduli space of the Calabi-Yau manifold $X, T^{*} \mathcal{M}_{c}(X) \times T^{*} \mathcal{M}_{\mathcal{K}}(X)$, cf. [29]. Since the moduli space itself is a Kähler space, this result agrees with the general analysis of $\mathcal{N}=(0,4)$ supersymmetric sigma-models. According to [32], $\mathcal{N}=(0,4)$ supersymmetric sigma-model is based on a target space which has three covariantly constant (with respect to $\nabla_{+}$) complex structures which obey the quaternionic algebra:

$$
J_{r} J_{s}=-\delta_{r s}+f_{r s}^{t} J_{t}
$$


Another interesting feature that we expect to see in this $\mathcal{N}=(0,4)$ theory is $S L(2, \mathbb{R})$ symmetry of classical Type IIB supergravity. Apart from $\varphi, l, r_{i}$ and $t_{i}$, all the scalar fields listed above are singlets with respect to this symmetry. The complex scalar $\lambda=l+i e^{-\varphi}$ transforms as (6.5) under $S L(2, \mathbb{R})$ duality transformation, with $a, b, c$ and $d$ real numbers obeying $a d-b c=1$. The doublet of real scalar fields $\left(r_{i}, t_{i}\right)$ transforms as a vector under the general $S L(2, \mathbb{R})$ transformation. In other words, only the scalar multiplets $\Sigma_{i}$ transform non-trivially under this symmetry, while all the other fields, including the supergravity itself, are $S L(2, \mathbb{R})$-singlets.

Finally, we remark that from T-duality with Type IIA string theory on a Calabi-Yau four-fold we expect an anomaly similar to (3.2) in Type IIB compactification on a CalabiYau four-fold. Recall that due to the global anomaly (3.2), in Type IIA vacuum we had to include $N=\frac{\chi}{24}$ fundamental strings filling two-dimensional space-time to cancel the tadpole. Under a T-duality in one of the space-time directions the winding modes of these strings transform into $\frac{\chi}{24}$ momentum modes:

$$
P_{+}-P_{-}=\frac{\chi}{24}
$$

in the Type IIB vacuum corresponding to compactification on the same Calabi-Yau fourfold $X$. Here, for the sake of simplicity, we assumed that there are no background fluxes. One can interpret $(6.19)$ as the difference in the zero-point energy of the left-moving and the right-moving Kaluza-Klein modes [31]. In order to see this, we note that a free boson on a circle has vacuum energy $-\frac{1}{24}$ and a periodic fermion has vacuum energy $+\frac{1}{24}$. Therefore, due to $\mathcal{N}=(0,4)$ supersymmetry, in the right sector bosonic and fermionic contributions cancel each other, i.e. $P_{+}=0$. In the left sector we have $48+6 h^{1,1}+6 h^{3,1}-2 h^{2,1}$ bosonic modes corresponding to the fields $\phi_{i}, \bar{\phi}_{\bar{i}}, \check{s}_{j}, r_{j}, t_{j}, u_{i}, q, \bar{q}, \varphi$ and $l$ along with $n_{-}=4 h^{1,1}$ fermionic modes, $c f$. (6.17). Hence, the total vacuum momentum in the left sector is non-zero and is given by the following formula:

$$
P_{-}=\frac{1}{24}\left(48+6 h^{1,1}+6 h^{3,1}-6 h^{2,1}\right)
$$

Using $P_{+}=0$ and the explicit expression (2.2) for the Euler number, one can easily obtain the formula (6.19). 


\section{Superspace Formulation of $\mathcal{N}=(0,4)$ Dilaton Supergravity}

In this section we construct $\mathcal{N}=(0,4)$ dilaton supergravity that arise, for example, in Type IIB superstring compactification on Calabi-Yau four-folds. As we demonstrated in the previous section such a theory has a number of distinct features which are absent in the existing superspace formulations of two-dimensional $\mathcal{N}=(0,4)$ supergravities. Namely, unlike the standard formulations with gauged $S U(2) R$-symmetry [28,29], new supergravity does not have a gauged symmetry and the supergravity multiplet contains a real dilaton field $\mathcal{V}$. Below we present a superspace construction of this theory obtained via de-gauging $S U(2)$ symmetry.

First let us remind that gauged $\mathcal{N}=(0,4)$ supergravity is defined in superspace by the following set of constraints [33]:

$$
\begin{gathered}
{\left[\nabla_{+i}, \nabla_{+j}\right\}=0, \quad\left[\nabla_{+i}, \nabla_{\dot{+}}{ }^{j}\right\}=i 2 \delta_{i}^{j} \nabla_{\ddagger},} \\
{\left[\nabla_{+i}, \nabla_{\ddagger}\right\}=0 \quad, \quad\left[\nabla_{+i}, \nabla_{=}\right\}=-i\left[\bar{\Sigma}^{+}{ }_{i} \mathcal{X}-\bar{\Sigma}^{+}{ }_{j} \mathcal{Y}_{i}{ }^{j}\right]} \\
{\left[\nabla_{\ddagger}, \nabla_{=}\right\}=-\frac{1}{2}\left[\Sigma^{+i} \nabla_{+i}+\bar{\Sigma}^{+}{ }_{i} \nabla_{\dot{+}}{ }^{i}+\mathcal{R} \mathcal{X}+i \mathcal{F}_{i}{ }^{j} \mathcal{Y}_{j}{ }^{i}\right]}
\end{gathered}
$$

on the covariant derivatives $\nabla_{A} \equiv\left(\nabla_{+i}, \nabla_{\dot{+}}^{i}, \nabla_{\ddagger}, \nabla_{=}\right)$:

$$
\nabla_{A}=E_{A}{ }^{B} D_{B}+\Lambda_{A} \mathcal{X}+i \mathcal{A}_{A i}{ }^{j} \mathcal{Y}_{j}{ }^{i}
$$

Here $E_{A}{ }^{B}$ is the supervielbein, and $\mathcal{X}$ and $\mathcal{Y}_{j}^{i}$ are the Lorentz and $S U(2)$ symmetry generators, respectively. The superfield $\mathcal{A}_{A i}{ }^{j}$ is $S U(2)$ gauge connection, while $\Lambda_{A}$ stands for the Lorentz spin-connection. We write [ , \} for the graded (anti-)commutator. Finally, $D_{A}$ denotes the flat space fermi and bose derivatives $D_{A} \equiv\left(\bar{D}_{+i}, D_{+}{ }^{i}, \partial_{\neq}, \partial_{=}\right)$.

The Lorentz generators act on $\nabla_{A}$ as follows:

$$
\begin{gathered}
{\left[\mathcal{X}, \nabla_{+i}\right\}=\frac{1}{2} \nabla_{+i} \quad, \quad\left[\mathcal{X}, \nabla_{\dot{+}}{ }^{i}\right\}=\frac{1}{2} \nabla_{\dot{+}}{ }^{i},} \\
{\left[\mathcal{X}, \nabla_{\ddagger}\right\}=\nabla_{\ddagger} \quad, \quad\left[\mathcal{X}, \nabla_{=}\right\}=-\nabla_{=} .}
\end{gathered}
$$

Similarly, for the action of $S U(2)$ gauge symmetry generators we have:

$$
\begin{gathered}
{\left[\mathcal{Y}_{j}{ }^{k}, \nabla_{+i}\right\}=\delta_{i}^{k} \nabla_{+j}-\frac{1}{2} \delta_{j}^{k} \nabla_{+i},} \\
{\left[\mathcal{Y}_{j}{ }^{k}, \nabla_{\dot{+}}{ }^{i}\right\}=-\delta_{j}^{i} \nabla_{\dot{+}}{ }^{k}+\frac{1}{2} \delta_{j}^{k} \nabla_{\dot{+}}{ }^{i},}
\end{gathered}
$$




$$
\left[\mathcal{Y}_{j}^{k}, \nabla_{\ddagger}\right\}=0 \quad, \quad\left[\mathcal{Y}_{j}^{k}, \nabla_{=}\right\}=0
$$

The constraints (7.1) lead to a set of Bianchi identities that are solved if:

$$
\begin{gathered}
\nabla_{\dot{+}}{ }^{i} \bar{\Sigma}^{+j}=0 \quad, \quad \nabla_{+i} \Sigma^{+j}=\frac{1}{2} \delta_{i}^{j} \mathcal{R}+i \mathcal{F}_{i}{ }^{j} \\
\nabla_{+i} \mathcal{R}=i 2 \nabla_{\ddagger} \bar{\Sigma}^{+}{ }_{i} \quad, \quad \nabla_{+i} \mathcal{F}_{j}{ }^{k}=-2 \delta_{i}^{k} \nabla_{\ddagger} \bar{\Sigma}^{+}{ }_{j}+\delta_{j}^{k} \nabla_{\ddagger} \bar{\Sigma}^{+}{ }_{i} .
\end{gathered}
$$

The first step in obtaining two-dimensional $\mathcal{N}=(0,4)$ supergravity theory that does not contain a gauged $S U(2)$ is to note that the covariant derivative in $(7.2)$ can be split as:

$$
\nabla_{A}=\widehat{\nabla}_{A}+i \mathcal{A}_{A k}^{l} \mathcal{Y}_{l}^{k}
$$

Since the superspace covariant derivative $\widehat{\nabla}_{A}$ does not contain the $S U(2)$ connection nor the generator, it can not describe two-dimensional $\mathcal{N}=(0,4)$ supergravity with gauged $S U(2)$ symmetry. We next use (7.3) to derive the form of the commutator algebra for the $\widehat{\nabla}_{A}$ operators.

A straightforward set of calculations leads to:

$$
\begin{gathered}
{\left[\widehat{\nabla}_{+i}, \widehat{\nabla}_{+j}\right\}=-i\left[\mathcal{A}_{+i j}{ }^{k}+\mathcal{A}_{+j i}{ }^{k}\right] \widehat{\nabla}_{+k},} \\
{\left[\widehat{\nabla}_{+i}, \widehat{\nabla}_{\dot{+}}{ }^{j}\right\}=i 2 \delta_{i}^{j} \widehat{\nabla}_{\ddagger}+i \overline{\mathcal{A}}_{+}{ }_{i}{ }^{k} \widehat{\nabla}_{+k}+i \mathcal{A}_{+i k}{ }^{j} \widehat{\nabla}_{\dot{+}}{ }^{k},} \\
{\left[\widehat{\nabla}_{+i}, \widehat{\nabla}_{\ddagger}\right\}=i \mathcal{A}_{\neq i}{ }^{k} \widehat{\nabla}_{+k} \quad, \quad\left[\widehat{\nabla}_{+i}, \widehat{\nabla}_{=}\right\}=i \mathcal{A}_{=i}{ }^{k} \widehat{\nabla}_{+k}-i \Sigma^{+}{ }_{i} \mathcal{X},} \\
{\left[\widehat{\nabla}_{\ddagger}, \widehat{\nabla}_{=}\right\}=-\frac{1}{2}\left[\Sigma^{+i} \widehat{\nabla}_{+i}+\bar{\Sigma}^{+}{ }_{i} \widehat{\nabla}_{\dot{+}}{ }^{i}+\mathcal{R} \mathcal{X}\right]}
\end{gathered}
$$

where the connection superfields now explicitly appear on the right-hand side of the equations. The leading component of $\mathcal{A}_{+i j}{ }^{k}$ is a component of the gauged supergravity multiplet that could be eliminated by a gauge transformation in the $S U(2)$ gauge-symmetric phase.

At this stage, we have completed half of the de-gauging process. The second half consists of specifying the spinorial $S U(2)$ connections in terms of some components of another (matter) multiplet that is consistent with the two-dimensional $\mathcal{N}=(0,4)$ supergravity theory. For this purpose, we introduce the second of the four distinct $\mathcal{N}=(0,4)$ scalar multiplets (SM-II) that were discussed in [29, 30]. In the case of rigid supersymmetry this multiplet is described by:

$$
D_{+i} \mathcal{V}=i \lambda^{-}{ }_{i} \quad, \quad \overline{\mathcal{V}}=\mathcal{V} \quad, \quad \varphi^{i}{ }_{i}=0 \quad,
$$




$$
\begin{gathered}
D_{+i} \varphi_{j}{ }^{k}=2 \delta_{i}^{k} \lambda^{-}{ }_{j}-\delta_{j}^{k} \lambda^{-}{ }_{i} \quad, \quad \bar{\varphi}_{j}{ }^{i}=\varphi_{i}{ }^{j} \quad, \\
D_{\dot{+}}{ }^{i} \lambda^{-}{ }_{j}=\delta_{j}^{i} \partial_{\ddagger} \mathcal{V}+i \partial_{\ddagger} \varphi_{j}{ }^{i} \quad, \quad D_{+i} \lambda^{-}{ }_{j}=0 .
\end{gathered}
$$

In the locally supersymmetric theory one has to replace $D_{\alpha}$ by $\nabla_{\alpha}$.

The triplet of spin- 0 fields $\varphi_{j}{ }^{i}$ can be "eaten" by the triplet of spin- 1 fields in the minimal $\mathcal{N}=(0,4)$ supergravity multiplet and thus become their longitudinal components via the usual Goldstone mechanism. This eliminates the local $S U(2)$ symmetry. The scalar and spinor field of the matter multiplet become the dilaton and dilatino.

We are thus led to conjecture that the new form of two-dimensional $\mathcal{N}=(0,4)$ supergravity with a component spectrum given by:

$$
\begin{array}{ll}
e_{\mu}{ }^{\nu} & \text { graviton, } \\
\psi_{\mu}{ }^{+i} & S U(2) \text {-doublet, gravitino, } \\
A_{\mu i}{ }^{j} & S U(2) \text {-triplet, vector auxiliary fields, } \\
\lambda^{-}{ }_{i} & S U(2) \text {-doublet, dilatino field and } \\
\mathcal{V} & \text { real dilaton field }
\end{array}
$$

may be constructed with (7.4) as its starting point. We note that the chirality of the dilatino is opposite to that of the gravitino.

In order to gain a control over the component field content of the theory, we must impose the following constraints:

$$
\begin{gathered}
\mathcal{A}_{+i j}{ }^{k}=\left[2 \lambda^{-}{ }_{j} \delta_{i}^{k}-\lambda^{-}{ }_{i} \delta_{j}^{k}\right], \\
\overline{\mathcal{A}}_{+{ }_{j}{ }^{k}{ }^{k}}=-\left[2 \bar{\lambda}^{-}{ }_{j} \delta_{i}^{k}-\bar{\lambda}^{-}{ }_{i} \delta_{j}^{k}\right]
\end{gathered}
$$

At lowest order in $\theta^{\alpha}$, the field $\mathcal{A}_{+i j}{ }^{k}$ is a component field that is absent in the $S U(2)$ gauge-symmetric phase of the theory; it can be set to zero in the Wess-Zumino gauge. On the other hand, when the $S U(2)$ symmetry is broken, part of this field becomes dynamical. In general, $\mathcal{A}_{+i j}{ }^{k}$ contains $S U(2)$ representations of spin- $\frac{1}{2}$ and spin- $\frac{3}{2}$. However, the above constraints eliminate the pure spin- $\frac{3}{2}$ representation of $S U(2)$.

With this result substituted into (7.4),

$$
\begin{gathered}
{\left[\widehat{\nabla}_{+i}, \widehat{\nabla}_{+j}\right\}=-i\left[\lambda^{-}{ }_{i} \widehat{\nabla}_{+j}+\lambda^{-}{ }_{j} \widehat{\nabla}_{+i}\right]} \\
{\left[\widehat{\nabla}_{+i}, \widehat{\nabla}_{\dot{+}}{ }^{j}\right\}=i 2 \delta_{i}^{j} \widehat{\nabla}_{\ddagger}+i\left[2 \bar{\lambda}^{-k} \delta_{i}^{j}-\bar{\lambda}^{-j} \delta_{i}^{k}\right] \widehat{\nabla}_{+k}+i\left[2 \lambda^{-}{ }_{k} \delta_{i}^{j}-\lambda^{-}{ }_{i} \delta_{k}^{j}\right] \widehat{\nabla}_{+}{ }^{k}} \\
{\left[\widehat{\nabla}_{+i}, \widehat{\nabla}_{\ddagger}\right\}=i \mathcal{A}_{\ddagger i}{ }^{k} \widehat{\nabla}_{+k} \quad, \quad\left[\widehat{\nabla}_{+i}, \widehat{\nabla}_{=}\right\}=i \mathcal{A}_{=i}{ }^{k} \widehat{\nabla}_{+k}-i \Sigma^{+}{ }_{i} \mathcal{X}}
\end{gathered}
$$




$$
\left[\widehat{\nabla}_{\ddagger}, \widehat{\nabla}_{=}\right\}=-\frac{1}{2}\left[\Sigma^{+i} \widehat{\nabla}_{+i}+\bar{\Sigma}_{i}^{+} \widehat{\nabla}_{\dot{+}}^{i}+\mathcal{R} \mathcal{X}\right]
$$

we can calculate the Bianchi identities:

$$
\begin{aligned}
& {\left[\left[\widehat{\nabla}_{+i}, \widehat{\nabla}_{+j}\right\}, \widehat{\nabla}_{+k}\right\}+\left[\left[\widehat{\nabla}_{+k}, \widehat{\nabla}_{+i}\right\}, \widehat{\nabla}_{+j}\right\}+\left[\left[\widehat{\nabla}_{+j}, \widehat{\nabla}_{+k}\right\}, \widehat{\nabla}_{+i}\right\}=0} \\
& {\left[\left[\widehat{\nabla}_{+i}, \widehat{\nabla}_{+j}\right\}, \widehat{\nabla}_{+}{ }^{k}\right\}+\left[\left[\widehat{\nabla}_{+i}, \widehat{\nabla}_{\dot{+}}{ }^{k}\right\}, \widehat{\nabla}_{+j}\right\}+\left[\left[\widehat{\nabla}_{+j}, \widehat{\nabla}_{\dot{+}}{ }^{k}\right\}, \widehat{\nabla}_{+i}\right\}=0}
\end{aligned}
$$

These will be satisfied if:

$$
\begin{gathered}
\widehat{\nabla}_{+i} \lambda^{-}{ }_{j}=-\lambda^{-}{ }_{i} \lambda^{-}{ }_{j}, \\
\widehat{\nabla}_{+i} \bar{\lambda}^{-j}=i \lambda^{-}{ }_{i} \bar{\lambda}^{-j}-i \mathcal{A}_{\neq i}{ }^{j}+\delta_{j}{ }_{j} \widehat{\nabla}_{\ddagger} \mathcal{V} \quad, \text { etc. }
\end{gathered}
$$

Let us now briefly comment on density projectors in the new $\mathcal{N}=(0,4)$ dilaton supergravity theory. For a general superspace Lagrangian $\mathcal{L}$, the component action of $\mathcal{N}=(0,4)$ gauged supergravity can be obtained by means of the following projection formula:

$$
\begin{gathered}
\int d^{2} x d^{2} \theta^{\ddagger} d^{2} \bar{\theta}^{\ddagger} E^{-1} \mathcal{L}=\frac{1}{2} \int d^{2} x d^{2} \theta^{\ddagger} \mathcal{E}^{-1}\left[\frac{1}{2} C_{i j} \widehat{\nabla}_{\dot{+}}{ }^{i} \widehat{\nabla}_{\dot{+}}{ }^{j}\right] \mathcal{L} \mid+ \\
+\frac{1}{2} \int d^{2} x d^{2} \bar{\theta}^{\ddagger} \overline{\mathcal{E}}^{-1}\left[\frac{1}{2} C^{i j} \widehat{\nabla}_{+i} \widehat{\nabla}_{+j}\right] \mathcal{L} \mid
\end{gathered}
$$

where the corresponding chiral and anti-chiral density projector formulas look like:

$$
\begin{aligned}
\int d^{2} x d^{2} \theta^{\ddagger} \mathcal{E}^{-1} \mathcal{L} \mid & =i \int d^{2} x\left[\frac{1}{2} e^{-1} C^{i j}\left(\widehat{\nabla}_{+i}+i 4 e \bar{\psi}_{\ddagger}{ }^{+}{ }_{i}\right)\right] \widehat{\nabla}_{+j} \mathcal{L} \mid \\
\int d^{2} x d^{2} \bar{\theta}^{\ddagger} \overline{\mathcal{E}}^{-1} \mathcal{L} \mid & =i \int d^{2} x\left[\frac{1}{2} e^{-1} C_{i j}\left(\widehat{\nabla}_{\dot{+}}{ }^{i}+i 4 e \psi_{\ddagger}{ }^{+i}\right)\right] \widehat{\nabla}_{\dot{+}}{ }^{j} \mathcal{L} \mid
\end{aligned}
$$

Similar formulas also hold in the new two-dimensional $\mathcal{N}=(0,4)$ dilaton supergravity. The explicit expressions for the density projectors can be obtained by a straightforward but tedious computation substituting $(7.3)$ into $(7.6)$.

"I found a way to make it work."

Stanislaw Ulam

\section{Acknowledgments}

We are grateful to Marc Grisaru, Martin Roček and John H. Schwarz for useful discussions. The research of S.J.G. is supported by the NSF grant No PHY-98-02551; S.G. is supported in part by the Caltech Discovery Fund, grant RFBR No 98-02-16575 and Russian President's grant No 96-15-96939. The work of E.W. is supported in part by NSF Grant PHY-9513835 and the Caltech Discovery Fund. 


\section{Appendix A. World-Sheet Calculation of Type IIA String Amplitudes}

Consider compactification of Type IIA string theory on a Calabi-Yau four-fold $X$. Let us further assume that there are no background Ramond-Ramond fluxes, so that two-dimensional space-time is flat. From the world-sheet viewpoint, this compactification corresponds to adjoining $c=(12,12) \mathcal{N}=(2,2)$ superconformal field theory (SCFT) to free conformal theory with central charge $c=(3,3)$ that is responsible for the two-dimensional space-time. It is the first part that will be interesting to us. Namely, we are going to show that two-point correlation function of vertex operators corresponding to chiral and twisted chiral superfields is zero, i.e. that the Zamolodchikov metric on the moduli space of Calabi-Yau four-folds is block diagonal (3.8).

$\mathcal{N}=(2,2)$ superconformal algebra consists of two $\mathcal{N}=2$ supervirasoro algebras one left-moving and one right-moving - each generated by an energy-momentum tensor $T$, a current $J$ and two weight $3 / 2$ supercurrents $G^{ \pm}$with $J$-charge $Q= \pm 1$. Recall that in a Kaluza-Klein reduction two-dimensional chiral superfields come from harmonic $(3,1)$-forms on $X$, while twisted chiral superfields correspond to harmonic $(1,1)$-forms. Similar to the three-fold case, we identify these fields with marginal operators in $(c, c)$ and $(a, c)$ multiplets, respectively. Let us call this operators $\Phi_{(1,1)}$ and $\Phi_{(-1,1)}$. They are neutral, $(Q, \bar{Q})=(0,0)$, and have conformal weight $1 / 2$. The lowest components of (anti-)chiral multiplets must satisfy $2 h=Q$ and $2 \bar{h}=\bar{Q}$, so $\Phi_{(1,1)}$ and $\Phi_{(-1,1)}$ are not the lowest components in the corresponding multiplets. They can be obtained in the operator product expansion of the supercurrents with operators $\Psi$ :

$$
2 G^{\mp}(w, \bar{w}) \cdot \Psi_{( \pm 1,1)}(z, \bar{z})=\frac{1}{w-z} \Phi_{( \pm 1,1)}(z, \bar{z})+\mathrm{reg}
$$

where "reg" stands for the regular part. Another operator product expansion that will be useful to us is the following:

$$
2 G^{\mp}(w, \bar{w}) \cdot \Phi_{( \pm 1,1)}(z, \bar{z})=\operatorname{reg}
$$

Now we are ready to demonstrate (3.8). Consider a matrix element of the target space metric $\mathcal{G}_{\phi_{i} \bar{\sigma}_{j}}$ that mixes $(1,1)$ and $(3,1)$ moduli:

$$
\begin{gathered}
\frac{\mathcal{G}_{\phi_{i} \bar{\sigma}_{j}}}{\left|z-z^{\prime}\right|^{4}}=\left\langle\Phi_{(1,1)}^{\phi_{i}}(z, \bar{z}) \cdot \Phi_{(1,-1)}^{\bar{\sigma}_{j}}\left(z^{\prime}, \bar{z}^{\prime}\right)\right\rangle= \\
=\oint \frac{d w}{2 \pi i}\left\langle 2 G^{-}(w, \bar{w}) \cdot \Psi_{(1,1)}^{\phi_{i}}(z, \bar{z}) \cdot \Phi_{(1,-1)}^{\bar{\sigma}_{j}}\left(z^{\prime}, \bar{z}^{\prime}\right)\right\rangle=0
\end{gathered}
$$


In the last equality we used the fact (A.2) that the operator product of $G^{-}(w, \bar{w})$ and $\Phi_{(1,-1)}^{\bar{\sigma}_{j}}\left(z^{\prime}, \bar{z}^{\prime}\right)$ has no singularity as $w \rightarrow z^{\prime}$.

One might think that $\mathcal{G}_{\phi_{i} \sigma_{j}}$ would be non-zero since the corresponding OPE has a singular part:

$$
2 G^{ \pm}(w, \bar{w}) \cdot \Phi_{( \pm 1,1)}(z, \bar{z})=\frac{\partial}{\partial z}\left(\frac{\Psi_{( \pm 1,1)}(z, \bar{z})}{w-z}\right)+\operatorname{reg}
$$

However, repeating the above arguments in the right sector one can easily see that $\mathcal{G}_{\phi_{i} \sigma_{j}}$ is also zero. In fact, OPE is singular in both left and right sectors only for $\left\langle\Phi_{(1,1)}^{\phi_{i}} \cdot \bar{\Phi}_{(-1,-1)}^{\bar{\phi}_{\bar{j}}}\right\rangle$

and $\left\langle\Phi_{(-1,1)}^{\sigma_{i}} \cdot \bar{\Phi}_{(1,-1)}^{\bar{\sigma}_{j}}\right\rangle$ which correspond to the metric $\mathcal{G}_{\phi_{i} \bar{\phi}_{\bar{j}}}$ for chiral multiplets and the metric $\mathcal{G}_{\sigma_{i} \bar{\sigma}_{j}}$ for the twisted chiral multiplets, respectively. Therefore, we conclude that the target space is locally a product of the manifold $\mathcal{M}_{c}(X)$ parametrized by the chiral fields $\phi_{i}$ and the manifold $\mathcal{M}_{\mathcal{K}}(X)$ spanned by the twisted chiral fields $\sigma_{j}$.

\section{Appendix B. Extra derivative constraints arising from de-gauging $\mathcal{N}=(2,2)$ non-minimal supergravity}

In this appendix we collect some more technical formulas that arise in the construction of $\mathcal{N}=(2,2)$ dilaton supergravity via de-gauging $U(1)_{A} \otimes U(1)_{V}$ non-minimal supergravity. Consistency of the de-gauging procedure requires that all terms in (4.8) with gauge symmetry generators $\mathcal{Y}$ and $\mathcal{Y}^{\prime}$ vanish, so that the commutator algebra of the new covariant derivative $\widehat{\nabla}_{\alpha}$ has the form (4.12), e.g.:

$$
\begin{gathered}
\left\{\widehat{\nabla}_{+}, \widehat{\nabla}_{+}\right\}=\left\{\nabla_{+}, \nabla_{+}\right\}-2\left\{\nabla_{+}, \lambda_{+} \mathcal{Y}+\widetilde{\lambda}_{+} \mathcal{Y}^{\prime}\right\}= \\
=-2\left(\nabla_{+} \lambda_{+}\right) \mathcal{Y}-2\left(\nabla_{+} \widetilde{\lambda}_{+}\right) \mathcal{Y}^{\prime}+i \lambda_{+} \nabla_{+}+i \widetilde{\lambda}_{+} \nabla_{+}= \\
=i\left(\lambda_{+}+\widetilde{\lambda}_{+}\right)\left(\widehat{\nabla}_{+}+\lambda_{+} \mathcal{Y}+\widetilde{\lambda}_{+} \mathcal{Y}^{\prime}\right)-2\left(\nabla_{+} \lambda_{+}\right) \mathcal{Y}-2\left(\nabla_{+} \widetilde{\lambda}_{+}\right) \mathcal{Y}^{\prime}= \\
=i\left(\lambda_{+}+\widetilde{\lambda}_{+}\right) \widehat{\nabla}_{+}+\left[-2\left(\nabla_{+} \lambda_{+}\right)+i \widetilde{\lambda}_{+} \lambda_{+}\right] \mathcal{Y}+\left[-2\left(\nabla_{+} \widetilde{\lambda}_{+}\right)+i \lambda_{+} \widetilde{\lambda}_{+}\right] \mathcal{Y}^{\prime}= \\
=i\left(\lambda_{+}+\widetilde{\lambda}_{+}\right) \widehat{\nabla}_{+}+\left[-2\left(\widehat{\nabla}_{+} \lambda_{+}\right)+i \widetilde{\lambda}_{+} \lambda_{+}+i \widetilde{\lambda}_{+} \lambda_{+}\right] \mathcal{Y}+\left[-2\left(\widehat{\nabla}_{+} \tilde{\lambda}_{+}\right)+i \lambda_{+} \widetilde{\lambda}_{+}+i \lambda_{+} \widetilde{\lambda}_{+}\right] \mathcal{Y}^{\prime}= \\
=i\left(\lambda_{+}+\widetilde{\lambda}_{+}\right) \widehat{\nabla}_{+}+\left[-2\left(\widehat{\nabla}_{+} \lambda_{+}\right)+2 i \widetilde{\lambda}_{+} \lambda_{+}\right] \mathcal{Y}+\left[-2\left(\widehat{\nabla}_{+} \widetilde{\lambda}_{+}\right)+2 i \lambda_{+} \widetilde{\lambda}_{+}\right] \mathcal{Y}^{\prime}
\end{gathered}
$$

Therefore, we obtain the following commutation relation:

$$
\left\{\widehat{\nabla}_{+}, \widehat{\nabla}_{+}\right\}=i\left(\lambda_{+}+\widetilde{\lambda}_{+}\right) \widehat{\nabla}_{+}
$$


plus two constraints (Jacobi identities):

$$
\left(\widehat{\nabla}_{+} \lambda_{+}\right)-i \widetilde{\lambda}_{+} \lambda_{+}=0 \quad, \quad\left(\widehat{\nabla}_{+} \widetilde{\lambda}_{+}\right)-i \lambda_{+} \widetilde{\lambda}_{+}=0 .
$$

Similar calculations lead to the following set of constraints:

$$
\begin{aligned}
& \widehat{\nabla}_{+} \lambda_{+}-i \widetilde{\lambda}_{+} \lambda_{+}=0, \quad, \quad \widehat{\nabla}_{+} \widetilde{\lambda}_{+}-i \lambda_{+} \widetilde{\lambda}_{+}=0, \\
& \widehat{\nabla}_{-} \lambda_{-}+i \widetilde{\lambda}_{-} \lambda_{-}=0, \quad, \quad \hat{\nabla}_{-} \tilde{\lambda}_{-}-i \lambda_{-} \tilde{\lambda}_{-}=0, \\
& \widehat{\nabla}_{+} \lambda_{-}+\widehat{\nabla}_{-} \lambda_{+}+i\left(\widetilde{\lambda}_{+} \lambda_{-}-\widetilde{\lambda}_{-} \lambda_{+}\right)=0, \\
& \widehat{\nabla}_{+} \tilde{\lambda}_{\dot{-}}+\widehat{\nabla}_{\dot{-}} \tilde{\lambda}_{+}+i\left(\lambda_{+} \tilde{\lambda}_{\dot{-}}-\lambda_{\dot{-}} \tilde{\lambda}_{+}\right)=0, \\
& \widehat{\nabla}_{+} \tilde{\lambda}_{-}+\widehat{\nabla}_{-} \tilde{\lambda}_{+}+2 i \widetilde{\lambda}_{+} \tilde{\lambda}_{-}-i\left(\lambda_{+} \tilde{\lambda}_{-}+\lambda_{-} \tilde{\lambda}_{+}\right)-\frac{i}{2} \bar{R}=0, \\
& \widehat{\nabla}_{+} \lambda_{\dot{-}}+\widehat{\nabla}_{\dot{-}} \lambda_{+}+2 i \lambda_{+} \lambda_{\dot{-}}-i\left(\widetilde{\lambda}_{+} \lambda_{\dot{-}}+\widetilde{\lambda}_{\dot{-}} \lambda_{+}\right)-\frac{i}{2} \bar{F}=0 \text {, } \\
& \widetilde{\lambda}_{\neq}=-i\left(\widehat{\nabla}_{+} \tilde{\lambda}_{\dot{+}}+\widehat{\nabla}_{\dot{+}} \widetilde{\lambda}_{+}\right)+2 \widetilde{\lambda}_{+} \widetilde{\lambda}_{\dot{+}}+\left(\lambda_{+} \widetilde{\lambda}_{\dot{+}}+\widetilde{\lambda}_{+} \lambda_{\dot{+}}\right) \text {, } \\
& \lambda_{\ddagger}=-i\left(\widehat{\nabla}_{+} \lambda_{\dot{+}}+\widehat{\nabla}_{\dot{+}} \lambda_{+}\right)+2 \lambda_{+} \lambda_{\dot{+}}+\left(\lambda_{+} \tilde{\lambda}_{\dot{+}}+\widetilde{\lambda}_{+} \lambda_{\dot{+}}\right) \text {, } \\
& \widetilde{\lambda}_{=}=-i\left(\widehat{\nabla}_{-} \tilde{\lambda}_{-}+\widehat{\nabla}_{-} \tilde{\lambda}_{-}\right)-2 \widetilde{\lambda}_{-} \tilde{\lambda}_{-}+\left(\lambda_{-} \tilde{\lambda}_{-}+\tilde{\lambda}_{-} \lambda_{-}\right) \text {, } \\
& \lambda_{=}=-i\left(\widehat{\nabla}_{-} \lambda_{\dot{-}}+\widehat{\nabla}_{\dot{-}} \lambda_{-}\right)+2 \lambda_{-} \lambda_{\dot{-}}-\left(\lambda_{-} \tilde{\lambda}_{\dot{-}}+\widetilde{\lambda}_{-} \lambda_{\dot{-}}\right)
\end{aligned}
$$

By virtue of the above relations, the Jacobi identities (4.13) are automatically satisfied. Furthermore, only half of the spinor fields are independent. In section 4 we disscuss two

natural solutions to these constraints: when either $\eta_{\alpha}$ or $\widetilde{\eta}_{\alpha}$ are put to zero. In both cases the remaining spinor superfields can be expressed in terms of an unconstrained real superfield.

\section{Appendix C. Components of covariant derivatives in $\mathcal{N}=(2,2)$ dilaton super- gravity}

The expressions for the covariant derivatives in $\mathcal{N}=(2,2)$ dilaton supergravity evaluated at $\theta=0$ are, $c f$. [16]:

$$
\begin{gathered}
\widehat{\nabla}_{\alpha} \mid=\partial_{\alpha} \\
\widehat{\nabla}_{\mu}\left|=\mathbf{D}_{\mu}+\psi_{\mu}^{\alpha} \widehat{\nabla}_{\alpha}\right|+\psi_{\mu}^{\dot{\alpha}} \widehat{\nabla}_{\dot{\alpha}} \mid=
\end{gathered}
$$




$$
=\mathbf{D}_{\mu}+\psi_{\mu}^{\alpha} \partial_{\alpha}+\psi_{\mu}^{\dot{\alpha}} \partial_{\dot{\alpha}}
$$

where $\mathbf{D}_{\mu}$ is the fully covariant gravitational derivative with the Lorentz connection $\omega_{\mu}=$ $\Lambda_{\mu} \mid$ that includes, in addition to the ordinary connection, extra terms that are bilinear in the gravitini $\psi_{\mu}^{\alpha}, \psi_{\mu}^{\dot{\alpha}}$. Specifically, $\mathbf{D}_{\mu}$ is defined to be 11 .

$$
\mathbf{D}_{\mu}=e_{\mu}+\omega_{\mu} \mathcal{X}
$$

We also need expressions for the higher $\theta$ components of the covariant derivatives. The $\theta^{\alpha}$ component of $\widehat{\nabla}_{\beta}$ is defined by [16]:

$$
\widehat{\nabla}_{\alpha} \widehat{\nabla}_{\beta}\left|=\frac{1}{2}\left\{\widehat{\nabla}_{\alpha}, \widehat{\nabla}_{\beta}\right\}\right|
$$

while the $\theta^{\alpha}$ component of $\widehat{\nabla}_{\mu}$ is:

$$
\begin{gathered}
\widehat{\nabla}_{\alpha} \widehat{\nabla}_{\mu}\left|=\left[\widehat{\nabla}_{\alpha}, \widehat{\nabla}_{\mu}\right]\right|+\widehat{\nabla}_{\mu} \widehat{\nabla}_{\alpha} \mid= \\
=\left[\widehat{\nabla}_{\alpha}, \widehat{\nabla}_{\mu}\right]\left|+\mathbf{D}_{\mu} \widehat{\nabla}_{\alpha}\right|+\psi_{\mu}^{\beta} \widehat{\nabla}_{\beta} \widehat{\nabla}_{\alpha}\left|+\psi_{\mu}^{\dot{\beta}} \widehat{\nabla}_{\dot{\beta}} \widehat{\nabla}_{\alpha}\right|
\end{gathered}
$$

From (C.2) and (4.17) we obtain the following results:

$$
\begin{aligned}
& \widehat{\nabla}_{+} \widehat{\nabla}_{+}\left|=i \lambda_{+} \partial_{+} \quad, \quad \hat{\nabla}_{-} \hat{\nabla}_{-}\right|=i \lambda_{-} \partial_{-} \\
& \widehat{\nabla}_{\dot{+}} \widehat{\nabla}_{\dot{+}}\left|=-i \lambda_{\dot{+}} \partial_{\dot{+}} \quad, \quad \widehat{\nabla}_{\dot{-}} \hat{\nabla}_{\dot{-}}\right|=-i \lambda_{\dot{L}} \partial_{\dot{-}} \\
& \widehat{\nabla}_{+} \widehat{\nabla}_{-}\left|=-\frac{1}{4} \bar{H} \mathcal{X} \quad, \quad \widehat{\nabla}_{+} \hat{\nabla}_{-}\right|=-\frac{1}{4} \bar{G} \mathcal{X} \\
& \widehat{\nabla}_{\dot{+}} \widehat{\nabla}_{\dot{-}}\left|=-\frac{1}{4} H \mathcal{X} \quad, \quad \widehat{\nabla}_{\dot{+}} \widehat{\nabla}_{-}\right|=-\frac{1}{4} G \mathcal{X} \\
& \widehat{\nabla}_{+} \widehat{\nabla}_{\dot{+}} \mid=\frac{i}{2} \mathbf{D}_{\ddagger}+\frac{i}{2}\left(\psi_{\mp}^{+}+\lambda_{\dot{+}}\right) \partial_{+}+\frac{i}{2} \psi_{\ddagger}^{-} \partial_{-}+\frac{i}{2}\left(\psi_{\mp}^{\dot{+}}-\lambda_{+}\right) \partial_{\dot{+}}+\frac{i}{2} \psi_{\ddagger}^{\dot{\dot{q}}} \partial_{-} \\
& \widehat{\nabla}_{-} \widehat{\nabla}_{\dot{-}} \mid=\frac{i}{2} \mathbf{D}_{=}+\frac{i}{2} \psi_{=}^{+} \partial_{+}+\frac{i}{2}\left(\psi_{=}^{-}+\lambda_{\dot{-}}\right) \partial_{-}+\frac{i}{2} \psi_{=}^{\dot{+}} \partial_{\dot{+}}+\frac{i}{2}\left(\psi_{=}^{\dot{\doteq}}-\lambda_{-}\right) \partial_{-}
\end{aligned}
$$

and from (C.3) we derive the series of identities that appears below:

$$
\begin{gathered}
\widehat{\nabla}_{+} \widehat{\nabla}_{\ddagger}\left|=\mathbf{D}_{\ddagger} \widehat{\nabla}_{+}\right|+\frac{i}{2} \psi_{\ddagger}^{\dot{+}} \mathbf{D}_{\ddagger} \mid-\frac{1}{4}\left(\psi_{\ddagger}^{-} \bar{H}+\psi_{\ddagger}^{\dot{-}} \bar{G}\right) \mathcal{X}+\left[i \psi_{\ddagger}^{+} \lambda_{+}+\frac{i}{2} \psi_{\ddagger}^{\dot{+}}\left(\psi_{\ddagger}^{+}+\lambda_{\dot{+}}\right)-\right. \\
\left.-2 i \lambda_{+} \lambda_{\dot{+}}-\left(\widehat{\nabla}_{\dot{+}} \lambda_{+}\right)-\left(\widehat{\nabla}_{+} \lambda_{\dot{+}}\right)\right] \partial_{+}+\frac{i}{2} \psi_{\ddagger}^{\dot{+}} \psi_{\ddagger}^{-} \partial_{-}+\frac{i}{2} \psi_{\ddagger}^{\dot{+}}\left(\psi_{\ddagger}^{\dot{+}}-\lambda_{+}\right) \partial_{+}+\frac{i}{2} \psi_{\ddagger}^{\dot{+}} \psi_{\ddagger}^{\dot{亠}} \partial_{-}
\end{gathered}
$$

11 In the notations of [22] this would correspond to $\omega_{\mu}=\gamma_{\mu}=\varphi_{\mu}$. 


$$
\begin{aligned}
& \widehat{\nabla}_{-} \widehat{\nabla}_{\ddagger}\left|=\mathbf{D}_{\ddagger} \widehat{\nabla}_{-}\right|+\frac{i}{2} \psi_{=}^{\dot{\dot{D}}} \mathbf{D}_{=} \mid-\left[\frac{i}{2}\left(\widehat{\nabla}_{\dot{+}} \bar{R}\right)+\frac{i}{2}\left(\widehat{\nabla}_{+} F\right)+\frac{1}{2}\left(\lambda_{\dot{+}}+\frac{1}{2} \psi_{\mp}^{+}\right) H-\frac{1}{2}\left(\lambda_{+}-\frac{1}{2} \psi_{\mp}^{\dot{\dot{ }}}\right) G\right] \mathcal{X}+
\end{aligned}
$$

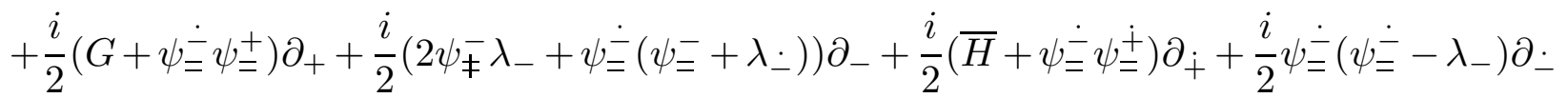

$$
\begin{aligned}
& \widehat{\nabla}_{\dot{+}} \widehat{\nabla}_{\ddagger}\left|=\mathbf{D}_{\ddagger} \widehat{\nabla}_{\dot{+}}\right|+\frac{i}{2} \psi_{\ddagger}^{\dot{+}} \mathbf{D}_{\ddagger} \mid-\frac{1}{4}\left(\psi_{\ddagger}^{-} G+\psi_{\ddagger}^{\dot{-}} H\right) \mathcal{X}+\frac{i}{2} \psi_{\ddagger}^{+}\left(\psi_{\ddagger}^{+}+\lambda_{\dot{+}}\right) \partial_{+}+\frac{i}{2} \psi_{\ddagger}^{+} \psi_{\ddagger}^{-} \partial_{-}+ \\
& +\left[2 i \lambda_{+} \lambda_{\dot{+}}+\left(\widehat{\nabla}_{\dot{+}} \lambda_{+}\right)+\left(\widehat{\nabla}_{+} \lambda_{\dot{+}}\right)+\frac{i}{2} \psi_{\neq}^{+}\left(\psi_{\neq}^{\dot{+}}-\lambda_{+}\right)-i \psi_{\neq}^{\dot{+}} \lambda_{\dot{+}}\right] \partial_{\dot{+}}+\frac{i}{2} \psi_{\neq}^{+} \psi_{\neq}^{\dot{\dot{j}}} \partial_{\dot{-}} \\
& \widehat{\nabla}_{\dot{-}} \widehat{\nabla}_{\ddagger}\left|=\mathbf{D}_{\ddagger} \widehat{\nabla}_{\dot{-}}\right|+\frac{i}{2} \psi_{\ddagger}^{-} \mathbf{D}_{=} \mid+\left[\frac{i}{2}\left(\widehat{\nabla}_{+} R\right)+\frac{i}{2}\left(\widehat{\nabla}_{\dot{+}} \bar{F}\right)-\frac{1}{2} \lambda_{+} H+\frac{1}{2} \lambda_{\dot{+}} \bar{G}-\frac{1}{4} \psi_{\ddagger}^{+} \bar{G}-\frac{1}{4} \psi_{\ddagger}^{\dot{+}} \bar{H}\right] \mathcal{X}+ \\
& +\frac{i}{2}\left(\psi_{\ddagger}^{-} \psi_{=}^{+}-H\right) \partial_{+}+\frac{i}{2} \psi_{\ddagger}^{-}\left(\psi_{=}^{-}+\lambda_{\dot{-}}\right) \partial_{-}+\frac{i}{2}\left(\psi_{\ddagger}^{-} \psi_{=}^{\dot{+}}-\bar{G}\right) \partial_{\dot{+}}+\frac{i}{2}\left(\psi_{\ddagger}^{-} \psi_{=}^{\dot{-}}-\psi_{\ddagger}^{-} \lambda_{-}-2 \psi_{\ddagger}^{\dot{-}} \lambda_{\dot{-}}\right) \partial_{\dot{-}} \\
& \widehat{\nabla}_{+} \widehat{\nabla}_{=}\left|=\mathbf{D}_{=} \widehat{\nabla}_{+}\right|+\frac{i}{2} \psi_{=}^{\dot{\perp}} \mathbf{D}_{=} \mid-\left[\frac{i}{2}\left(\widehat{\nabla}_{\dot{-}} \bar{R}\right)+\frac{i}{2}\left(\widehat{\nabla}_{-} \bar{F}\right)+\frac{1}{2} \lambda_{-} \bar{H}-\frac{1}{2} \lambda_{-} \bar{G}+\frac{1}{4} \psi_{=}^{+} \bar{H}+\frac{1}{4} \psi_{=}^{\dot{+}} G\right] \mathcal{X}+
\end{aligned}
$$

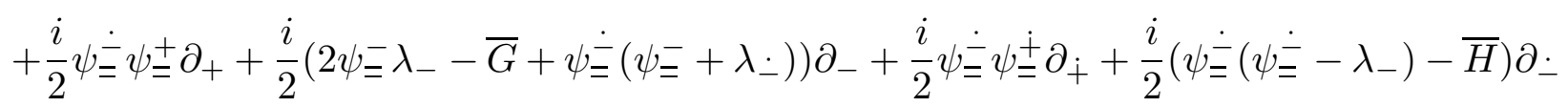

$$
\begin{aligned}
& \widehat{\nabla}_{-} \widehat{\nabla}_{=}\left|=\mathbf{D}_{=} \widehat{\nabla}_{-}\right|+\frac{i}{2} \psi_{=}^{\dot{\perp}} \mathbf{D}_{=} \mid-\frac{1}{4}\left(\psi_{=}^{+} \bar{H}+\psi_{=}^{\dot{ \pm}} G\right) \mathcal{X}+\frac{i}{2} \psi_{=}^{\dot{\perp}} \psi_{=}^{+} \partial_{+}+\left[i \psi_{=}^{-} \lambda_{-}+\right. \\
& \left.+\frac{i}{2} \psi_{=}^{\dot{\doteq}}\left(\psi_{=}^{-}+\lambda_{\dot{-}}\right)-2 i \lambda_{-} \lambda_{\dot{-}}-\left(\widehat{\nabla}_{\dot{-}} \lambda_{-}\right)-\left(\widehat{\nabla}_{-} \lambda_{\dot{-}}\right)\right] \partial_{-}+\frac{i}{2} \psi_{=}^{\dot{\doteq}} \psi_{=}^{\dot{+}} \partial_{\dot{+}}+\frac{i}{2} \psi_{=}^{\dot{\dot{ }}}\left(\psi_{=}^{\dot{\dot{\Xi}}}-\lambda_{-}\right) \partial_{-} \\
& \widehat{\nabla}_{\dot{+}} \widehat{\nabla}_{=}\left|=\mathbf{D}_{=} \widehat{\nabla}_{\dot{+}}\right|+\frac{i}{2} \psi_{=}^{+} \mathbf{D}_{\ddagger} \mid+\left[\frac{i}{2}\left(\widehat{\nabla}_{-} R\right)+\frac{i}{2}\left(\widehat{\nabla}_{-} F\right)-\frac{1}{2} \lambda_{-} H+\frac{1}{2} \lambda_{\dot{-}} G-\frac{1}{4} \psi_{=}^{-} G-\frac{1}{4} \psi_{=}^{\dot{\dot{ }}} H\right] \mathcal{X}+ \\
& +\frac{i}{2} \psi_{=}^{+}\left(\psi_{\neq}^{+}+\lambda_{\dot{+}}\right) \partial_{+}+\frac{i}{2}\left(H+\psi_{=}^{+} \psi_{\ddagger}^{-}\right) \partial_{-}+\frac{i}{2}\left(\psi_{=}^{+}\left(\psi_{\neq}^{\dot{+}}-\lambda_{+}\right)-2 \psi_{=}^{\dot{+}} \lambda_{\dot{+}}\right) \partial_{\dot{+}}+\frac{i}{2}\left(G+\psi_{=}^{+} \psi_{\ddagger}^{\dot{-}}\right) \partial_{-} \\
& \widehat{\nabla}_{\dot{-}} \widehat{\nabla}_{=}\left|=\mathbf{D}_{=} \widehat{\nabla}_{\dot{-}}\right|+\frac{i}{2} \psi_{=}^{-} \mathbf{D}_{=} \mid-\frac{1}{4}\left(\psi_{=}^{+} \bar{G}+\psi_{=}^{\dot{+}} H\right) \mathcal{X}+\frac{i}{2} \psi_{=}^{-} \psi_{=}^{+} \partial_{+}+\frac{i}{2} \psi_{=}^{-}\left(\psi_{=}^{-}+\lambda_{-}\right) \partial_{-}+ \\
& +\frac{i}{2} \psi_{=}^{=} \psi_{=}^{\dot{+}} \partial_{\dot{+}}++\left[2 i \lambda_{-} \lambda_{\dot{-}}+\left(\widehat{\nabla}_{\dot{-}} \lambda_{-}\right)+\left(\widehat{\nabla}_{-} \lambda_{\dot{-}}\right)+\frac{i}{2} \psi_{=}^{-}\left(\psi_{=}^{\dot{-}}-\lambda_{-}\right)-i \psi_{=}^{\dot{\Xi}} \lambda_{\dot{-}}\right] \partial_{-}
\end{aligned}
$$

where we also used the commutation relations (4.19).

Furthermore, the $\widehat{\nabla}_{\neq}$component of $\widehat{\nabla}_{=}$is given by:

$$
\widehat{\nabla}_{\ddagger} \widehat{\nabla}_{=}\left|=\widehat{\nabla}_{\ddagger}\right| \widehat{\nabla}_{=}\left|+\psi_{\neq}^{\alpha} \widehat{\nabla}_{\alpha} \widehat{\nabla}_{=}\right|+\psi_{\neq}^{\dot{\alpha}} \widehat{\nabla}_{\dot{\alpha}} \widehat{\nabla}_{=} \mid
$$

so that:

$$
\begin{gathered}
{\left[\widehat{\nabla}_{\ddagger}, \widehat{\nabla}_{=}\right]\left|=\left[\widehat{\nabla}_{\ddagger}\left|, \widehat{\nabla}_{=}\right|\right]+\psi_{\ddagger}^{\alpha} \widehat{\nabla}_{\alpha} \widehat{\nabla}_{=}\right|+\psi_{\ddagger}^{\dot{\alpha}} \widehat{\nabla}_{\dot{\alpha}} \widehat{\nabla}_{=}\left|-\psi_{=}^{\alpha} \widehat{\nabla}_{\alpha} \widehat{\nabla}_{\ddagger}\right|-\psi_{=}^{\dot{\alpha}} \widehat{\nabla}_{\dot{\alpha}} \widehat{\nabla}_{\ddagger} \mid=} \\
=\left[\mathbf{D}_{\ddagger}, \mathbf{D}_{=}\right]+\mathbf{D}_{[\mp}\left(\psi_{=]}^{\alpha} \partial_{\alpha}\right)+\mathbf{D}_{[\mp}\left(\psi_{=]}^{\dot{\alpha}} \partial_{\dot{\alpha}}\right)+\psi_{\ddagger}^{\alpha} \widehat{\nabla}_{\alpha} \widehat{\nabla}_{=}\left|+\psi_{\ddagger}^{\dot{\alpha}} \widehat{\nabla}_{\dot{\alpha}} \widehat{\nabla}_{=}\right|-\psi_{=}^{\alpha} \widehat{\nabla}_{\alpha} \widehat{\nabla}_{\ddagger}\left|-\psi_{=}^{\dot{\alpha}} \widehat{\nabla}_{\dot{\alpha}} \widehat{\nabla}_{\ddagger}\right|
\end{gathered}
$$


Substituting the above expressions for the components of $\widehat{\nabla}_{\alpha} \widehat{\nabla}_{\mu} \mid$ into $\left[\widehat{\nabla}_{\ddagger}, \widehat{\nabla}_{=}\right] \mid$, we get:

$$
\begin{aligned}
& {\left[\widehat{\nabla}_{\ddagger}, \widehat{\nabla}_{=}\right] \mid=\left[\mathbf{D}_{\ddagger}, \mathbf{D}_{=}\right]+\mathbf{D}_{[\ddagger}\left(\psi_{=]}^{\alpha} \partial_{\alpha}\right)+\mathbf{D}_{[\ddagger}\left(\psi_{=]}^{\dot{\alpha}} \partial_{\dot{\alpha}}\right)+} \\
& +\psi_{\ddagger}^{+}\left(\mathbf{D}_{=} \widehat{\nabla}_{\dot{+}}\left|+\frac{i}{2} \psi_{=}^{+} \mathbf{D}_{\ddagger}\right|+\left[\frac{i}{2}\left(\widehat{\nabla}_{-} R\right)+\frac{i}{2}\left(\widehat{\nabla}_{-} F\right)-\frac{1}{2} \lambda_{-} H+\frac{1}{2} \lambda_{-} G-\frac{1}{4} \psi_{=}^{-} G-\frac{1}{4} \psi_{=}^{\dot{\doteq}} H\right] \mathcal{X}+\right.
\end{aligned}
$$

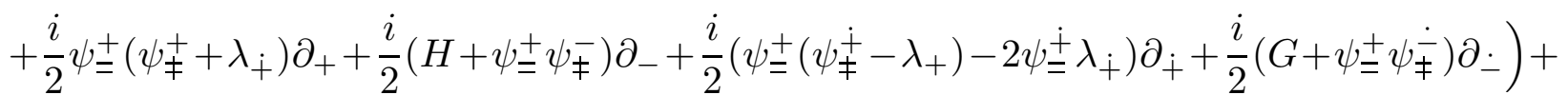

$$
\begin{aligned}
& +\psi_{\ddagger}^{-}\left(\mathbf{D}_{=} \widehat{\nabla}_{-}\left|+\frac{i}{2} \psi_{=}^{\dot{\doteq}} \mathbf{D}_{=}\right|-\frac{1}{4}\left(\psi_{=}^{+} \bar{H}+\psi_{=}^{\dot{ \pm}} G\right) \mathcal{X}+\frac{i}{2} \psi_{=}^{\dot{\doteq}} \psi_{=}^{+} \partial_{+}+\left[i \psi_{=}^{-} \lambda_{-}+\right.\right. \\
& \left.\left.+\frac{i}{2} \psi_{=}^{\dot{\dot{\Xi}}}\left(\psi_{=}^{-}+\lambda_{\dot{-}}\right)-2 i \lambda_{-} \lambda_{\dot{-}}-\left(\widehat{\nabla}_{\dot{-}} \lambda_{-}\right)-\left(\widehat{\nabla}_{-} \lambda_{\dot{-}}\right)\right] \partial_{-}+\frac{i}{2} \psi_{=}^{\dot{\dot{ }}} \psi_{=}^{\dot{+}} \partial_{\dot{+}}+\frac{i}{2} \psi_{=}^{\dot{\dot{ }}}\left(\psi_{=}^{\dot{\dot{\Xi}}}-\lambda_{-}\right) \partial_{-}\right)+ \\
& +\psi_{\mp}^{\dot{+}}\left(\mathbf{D}_{=} \widehat{\nabla}_{\dot{+}}\left|+\frac{i}{2} \psi_{=}^{+} \mathbf{D}_{\ddagger}\right|+\left[\frac{i}{2}\left(\widehat{\nabla}_{-} R\right)+\frac{i}{2}\left(\widehat{\nabla}_{-} F\right)-\frac{1}{2} \lambda_{-} H+\frac{1}{2} \lambda_{-} G-\frac{1}{4} \psi_{=}^{-} G-\frac{1}{4} \psi_{=}^{\dot{\dot{ }}} H\right] \mathcal{X}+\right. \\
& \left.+\frac{i}{2} \psi_{=}^{+}\left(\psi_{\neq}^{+}+\lambda_{\dot{+}}\right) \partial_{+}+\frac{i}{2}\left(H+\psi_{=}^{+} \psi_{\ddagger}^{-}\right) \partial_{-}+\frac{i}{2}\left(\psi_{=}^{+}\left(\psi_{\neq}^{\dot{+}}-\lambda_{+}\right)-2 \psi_{=}^{\dot{+}} \lambda_{\dot{+}}\right) \partial_{\dot{+}}+\frac{i}{2}\left(G+\psi_{=}^{+} \psi_{\ddagger}^{\dot{-}}\right) \partial_{-}\right)+ \\
& +\psi_{\mp}^{\dot{-}}\left(\mathbf{D}_{=} \widehat{\nabla}_{\dot{-}}\left|+\frac{i}{2} \psi_{=}^{-} \mathbf{D}_{=}\right|-\frac{1}{4}\left(\psi_{=}^{+} \bar{G}+\psi_{=}^{\dot{+}} H\right) \mathcal{X}+\frac{i}{2} \psi_{=}^{-} \psi_{=}^{+} \partial_{+}+\frac{i}{2} \psi_{=}^{-}\left(\psi_{=}^{-}+\lambda_{\dot{-}}\right) \partial_{-}+\right. \\
& \left.+\frac{i}{2} \psi_{=}^{-} \psi_{=}^{\dot{+}} \partial_{\dot{+}}+\left[2 i \lambda_{-} \lambda_{\dot{-}}+\left(\widehat{\nabla}_{\dot{-}} \lambda_{-}\right)+\left(\widehat{\nabla}_{-} \lambda_{\dot{-}}\right)+\frac{i}{2} \psi_{=}^{-}\left(\psi_{=}^{\dot{\Xi}}-\lambda_{-}\right)-i \psi_{=}^{\dot{\dot{\prime}}} \lambda_{\dot{-}}\right] \partial_{-}\right)- \\
& -\psi_{=}^{+}\left(\mathbf{D}_{\ddagger} \widehat{\nabla}_{+}\left|+\frac{i}{2} \psi_{\ddagger}^{\dot{+}} \mathbf{D}_{\ddagger}\right|-\frac{1}{4}\left(\psi_{\ddagger}^{-} \bar{H}+\psi_{\ddagger}^{\dot{-}} \bar{G}\right) \mathcal{X}+\left[i \psi_{\ddagger}^{+} \lambda_{+}+\frac{i}{2} \psi_{\ddagger}^{\dot{+}}\left(\psi_{\ddagger}^{+}+\lambda_{\dot{+}}\right)-\right.\right. \\
& \left.\left.-2 i \lambda_{+} \lambda_{\dot{+}}-\left(\widehat{\nabla}_{\dot{+}} \lambda_{+}\right)-\left(\widehat{\nabla}_{+} \lambda_{\dot{+}}\right)\right] \partial_{+}+\frac{i}{2} \psi_{\ddagger}^{\dot{+}} \psi_{\ddagger}^{-} \partial_{-}+\frac{i}{2} \psi_{\neq}^{\dot{+}}\left(\psi_{\neq}^{\dot{+}}-\lambda_{+}\right) \partial_{\dot{+}}+\frac{i}{2} \psi_{\neq}^{\dot{+}} \psi_{\ddagger}^{\dot{-}} \partial_{-}\right)- \\
& -\psi_{=}^{-}\left(\mathbf{D}_{\ddagger} \widehat{\nabla}_{-}\left|+\frac{i}{2} \psi_{=}^{\dot{\check{I}}} \mathbf{D}_{=}\right|-\left[\frac{i}{2}\left(\widehat{\nabla}_{\dot{+}} \bar{R}\right)+\frac{i}{2}\left(\widehat{\nabla}_{+} F\right)+\frac{1}{2}\left(\lambda_{\dot{+}}+\frac{1}{2} \psi_{\ddagger}^{+}\right) H-\frac{1}{2}\left(\lambda_{+}-\frac{1}{2} \psi_{\ddagger}^{\dot{+}}\right) G\right] \mathcal{X}+\right. \\
& \left.+\frac{i}{2}\left(G+\psi_{=}^{\dot{\dot{ }}} \psi_{=}^{+}\right) \partial_{+}+\frac{i}{2}\left(2 \psi_{\ddagger}^{-} \lambda_{-}+\psi_{=}^{\dot{\dot{\Xi}}}\left(\psi_{=}^{-}+\lambda_{\dot{-}}\right)\right) \partial_{-}+\frac{i}{2}\left(\bar{H}+\psi_{=}^{\dot{\dot{\Xi}}} \psi_{=}^{\dot{+}}\right) \partial_{+}+\frac{i}{2} \psi_{=}^{\dot{\doteq}}\left(\psi_{=}^{\dot{\dot{ }}}-\lambda_{-}\right) \partial_{-}\right)- \\
& -\psi_{=}^{\dot{+}}\left(\mathbf{D}_{\ddagger} \widehat{\nabla}_{\dot{+}}\left|+\frac{i}{2} \psi_{\ddagger}^{\dot{+}} \mathbf{D}_{\ddagger}\right|-\frac{1}{4}\left(\psi_{\ddagger}^{-} G+\psi_{\ddagger}^{\dot{-}} H\right) \mathcal{X}+\frac{i}{2} \psi_{\ddagger}^{+}\left(\psi_{\ddagger}^{+}+\lambda_{\dot{+}}\right) \partial_{+}+\frac{i}{2} \psi_{\ddagger}^{+} \psi_{\ddagger}^{-} \partial_{-}+\right. \\
& \left.+\left[2 i \lambda_{+} \lambda_{\dot{+}}+\left(\widehat{\nabla}_{\dot{+}} \lambda_{+}\right)+\left(\widehat{\nabla}_{+} \lambda_{\dot{+}}\right)+\frac{i}{2} \psi_{\neq}^{+}\left(\psi_{\neq}^{\dot{+}}-\lambda_{+}\right)-i \psi_{\neq}^{\dot{+}} \lambda_{\dot{+}}\right] \partial_{\dot{+}}+\frac{i}{2} \psi_{\ddagger}^{+} \psi_{\ddagger}^{\dot{-}} \partial_{-}\right)- \\
& -\psi_{=}^{\dot{\doteq}}\left(\mathbf{D}_{\ddagger} \widehat{\nabla}_{\dot{-}}\left|+\frac{i}{2} \psi_{\ddagger}^{-} \mathbf{D}_{=}\right|+\left[\frac{i}{2}\left(\widehat{\nabla}_{+} R\right)+\frac{i}{2}\left(\widehat{\nabla}_{\dot{+}} \bar{F}\right)-\frac{1}{2} \lambda_{+} H+\frac{1}{2} \lambda_{\dot{+}} \bar{G}-\frac{1}{4} \psi_{\ddagger}^{+} \bar{G}-\frac{1}{4} \psi_{\ddagger}^{\dot{+}} \bar{H}\right] \mathcal{X}+\right. \\
& \left.+\frac{i}{2}\left(\psi_{\ddagger}^{-} \psi_{=}^{+}-H\right) \partial_{+}+\frac{i}{2} \psi_{\ddagger}^{-}\left(\psi_{=}^{-}+\lambda_{\dot{-}}\right) \partial_{-}+\frac{i}{2}\left(\psi_{\ddagger}^{-} \psi_{=}^{\dot{+}}-\bar{G}\right) \partial_{\dot{+}}+\frac{i}{2}\left(\psi_{\ddagger}^{-} \psi_{=}^{\dot{-}}-\psi_{\ddagger}^{-} \lambda_{-}-2 \psi_{\ddagger}^{\dot{-}} \lambda_{\dot{-}}\right) \partial_{\dot{-}}\right)
\end{aligned}
$$

Now one can compare this huge formula with the leading component of the commutator $\left[\widehat{\nabla}_{\ddagger}, \widehat{\nabla}_{=}\right]$computed directly from the Bianchi identities (4.13):

$$
\left[\widehat{\nabla}_{\neq}, \widehat{\nabla}_{=}\right]=\left(\lambda_{+} S-\lambda_{+} \bar{S}+i\left(\widehat{\nabla}_{\dot{+}} S\right)+i\left(\widehat{\nabla}_{+} \bar{S}\right)\right) \mathcal{X}+
$$




$$
\begin{aligned}
& +\left(\frac{i}{2} \lambda_{\dot{-}} \bar{R}-\frac{i}{2} \lambda_{-} \bar{F}-\frac{1}{2}\left(\widehat{\nabla}_{\dot{-}} \bar{R}\right)-\frac{1}{2}\left(\widehat{\nabla}_{-} \bar{F}\right)\right) \widehat{\nabla}_{\dot{+}}+\left(\frac{i}{2} \lambda_{-} R-\frac{i}{2} \lambda_{\dot{-}} F+\frac{1}{2}\left(\widehat{\nabla}_{-} \bar{R}\right)+\frac{1}{2}\left(\widehat{\nabla}_{\dot{-}} F\right)\right) \widehat{\nabla}_{+}+ \\
& +\left(\frac{i}{2} \lambda_{\dot{+}} \bar{R}+\frac{i}{2} \lambda_{+} F-\frac{1}{2}\left(\widehat{\nabla}_{\dot{+}} \bar{R}\right)+\frac{1}{2}\left(\widehat{\nabla}_{+} F\right)\right) \widehat{\nabla}_{\dot{-}}+\left(\frac{i}{2} \lambda_{\dot{+}} \bar{F}+\frac{i}{2} \lambda_{+} R-\frac{1}{2}\left(\widehat{\nabla}_{\dot{+}} \bar{F}\right)+\frac{1}{2}\left(\widehat{\nabla}_{+} R\right)\right) \widehat{\nabla}_{-}
\end{aligned}
$$

where we denoted:

$$
S=\frac{i}{2}\left(\widehat{\nabla}_{-} \bar{R}\right)+\frac{i}{2}\left(\widehat{\nabla}_{-} \bar{F}\right)+\frac{1}{2} \lambda_{-} \cdot \bar{R}-\frac{1}{2} \lambda_{-} \bar{F}
$$

By comparing the coefficients of $\widehat{\nabla}_{+} \mid=\partial_{+}$in (C.4 and (C.5), for example, we find:

$$
\begin{aligned}
& \frac{i}{2} \lambda_{-} R-\frac{i}{2} \lambda_{-} F+\frac{1}{2}\left(\widehat{\nabla}_{-} \bar{R}\right)+\frac{1}{2}\left(\widehat{\nabla}_{-} F\right)=\mathbf{D}_{[\ddagger} \psi_{=]}^{+}+\frac{i}{2} \psi_{\ddagger}^{+} \psi_{=}^{+}\left(\psi_{\ddagger}^{+}+\lambda_{\dot{+}}\right)+ \\
& +\frac{i}{2} \psi_{\ddagger}^{-} \psi_{=}^{\dot{\dot{\Xi}}} \psi_{=}^{+}+\frac{i}{2} \psi_{\ddagger}^{\dot{+}} \psi_{=}^{+}\left(\psi_{\ddagger}^{+}+\lambda_{\dot{+}}\right)+\frac{i}{2} \psi_{\ddagger}^{\dot{-}} \psi_{=}^{-} \psi_{=}^{+}-\psi_{=}^{+}\left[i \psi_{\ddagger}^{+} \lambda_{+}+\frac{i}{2} \psi_{\ddagger}^{\dot{+}}\left(\psi_{\ddagger}^{+}+\lambda_{\dot{+}}\right)-2 i \lambda_{+} \lambda_{\dot{+}}-\right. \\
& \left.-\left(\widehat{\nabla}_{\dot{+}} \lambda_{+}\right)-\left(\widehat{\nabla}_{+} \lambda_{\dot{+}}\right)\right]-\frac{i}{2} \psi_{=}^{-}\left(G+\psi_{=}^{\dot{=}} \psi_{=}^{+}\right)-\frac{i}{2} \psi_{=}^{\dot{\dot{+}}} \psi_{\ddagger}^{+}\left(\psi_{\ddagger}^{+}+\lambda_{\dot{+}}\right)-\frac{i}{2} \psi_{=}^{\dot{\dot{ }}}\left(\psi_{\ddagger}^{-} \psi_{=}^{+}-H\right)
\end{aligned}
$$

In the same fashion we obtain the relations defining the other components of the superfields $R$ and $F$ :

$$
\begin{aligned}
& \frac{i}{2} \lambda_{\dot{+}} \bar{F}+\frac{i}{2} \lambda_{+} R-\frac{1}{2}\left(\widehat{\nabla}_{\dot{+}} \bar{F}\right)+\frac{1}{2}\left(\widehat{\nabla}_{+} R\right)=\mathbf{D}_{[\mp} \psi_{=]}^{-}+\frac{i}{2} \psi_{\ddagger}^{+}\left(H+\psi_{=}^{+} \psi_{\ddagger}^{-}\right)+\psi_{\ddagger}^{-}\left[i \psi_{=}^{-} \lambda_{-}+\right. \\
& \left.+\frac{i}{2} \psi_{=}^{\dot{\dot{ }}}\left(\psi_{=}^{-}+\lambda_{\dot{-}}\right)-2 i \lambda_{-} \lambda_{\dot{-}}-\left(\widehat{\nabla}_{\dot{-}} \lambda_{-}\right)-\left(\widehat{\nabla}_{-} \lambda_{\dot{-}}\right)\right]+\frac{i}{2} \psi_{\ddagger}^{\dot{+}}\left(H+\psi_{=}^{+} \psi_{\ddagger}^{-}\right)+\frac{i}{2} \psi_{\ddagger}^{\dot{-}} \psi_{=}^{-}\left(\psi_{=}^{-}+\lambda_{\dot{-}}\right)- \\
& -\frac{i}{2} \psi_{=}^{+} \psi_{\mp}^{\dot{+}} \psi_{\ddagger}^{-}-\frac{i}{2} \psi_{=}^{-}\left(2 \psi_{\mp}^{-} \lambda_{-}+\psi_{=}^{\dot{\dot{\Xi}}}\left(\psi_{=}^{-}+\lambda_{\dot{-}}\right)\right)-\frac{i}{2} \psi_{=}^{\dot{+}} \psi_{\ddagger}^{+} \psi_{\ddagger}^{-}-\frac{i}{2} \psi_{=}^{\dot{\dot{ }}} \psi_{\mp}^{-}\left(\psi_{=}^{-}+\lambda_{\dot{-}}\right) \\
& \frac{i}{2} \lambda_{-} \bar{R}-\frac{i}{2} \lambda_{-} \bar{F}-\frac{1}{2}\left(\widehat{\nabla}_{\dot{-}} \bar{R}\right)-\frac{1}{2}\left(\widehat{\nabla}_{-} \bar{F}\right)= \\
& =\mathbf{D}_{[\ddagger} \psi_{=]}^{\dot{+}}+\frac{i}{2} \psi_{\ddagger}^{+}\left(\psi_{=}^{+}\left(\psi_{\ddagger}^{\dot{+}}-\lambda_{+}\right)-2 \psi_{=}^{\dot{+}} \lambda_{\dot{+}}\right)+\frac{i}{2} \psi_{\ddagger}^{-} \psi_{=}^{\dot{\dot{ }}} \psi_{=}^{\dot{+}}+\frac{i}{2} \psi_{\ddagger}^{\dot{\dot{+}}}\left(\psi_{=}^{+}\left(\psi_{\ddagger}^{\dot{+}}-\lambda_{+}\right)-2 \psi_{=}^{\dot{+}} \lambda_{\dot{+}}\right)+ \\
& +\frac{i}{2} \psi_{\ddagger}^{\dot{-}} \psi_{=}^{-} \psi_{=}^{\dot{+}}-\frac{i}{2} \psi_{=}^{+} \psi_{\neq}^{\dot{+}}\left(\psi_{\neq}^{\dot{+}}-\lambda_{+}\right)-\frac{i}{2} \psi_{=}^{-}\left(\bar{H}+\psi_{=}^{\dot{\dot{\Xi}}} \psi_{=}^{\dot{+}}\right)-\psi_{=}^{\dot{+}}\left[2 i \lambda_{+} \lambda_{\dot{+}}+\left(\widehat{\nabla}_{\dot{+}} \lambda_{+}\right)+\right. \\
& \left.+\left(\widehat{\nabla}_{+} \lambda_{\dot{+}}\right)+\frac{i}{2} \psi_{\mp}^{+}\left(\psi_{\ddagger}^{\dot{+}}-\lambda_{+}\right)-i \psi_{\mp}^{\dot{+}} \lambda_{\dot{+}}\right]-\frac{i}{2} \psi_{=}^{\dot{\dot{\perp}}}\left(\psi_{\mp}^{-} \psi_{=}^{\dot{+}}-\bar{G}\right) \\
& \frac{i}{2} \lambda_{\dot{+}} \bar{R}+\frac{i}{2} \lambda_{+} F-\frac{1}{2}\left(\widehat{\nabla}_{\dot{+}} \bar{R}\right)+\frac{1}{2}\left(\widehat{\nabla}_{+} F\right)=
\end{aligned}
$$

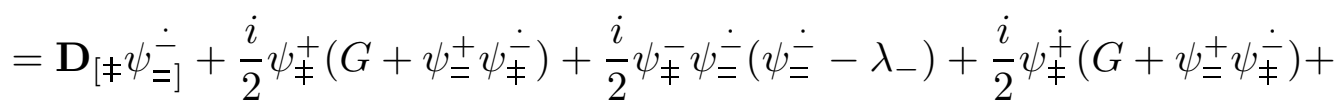

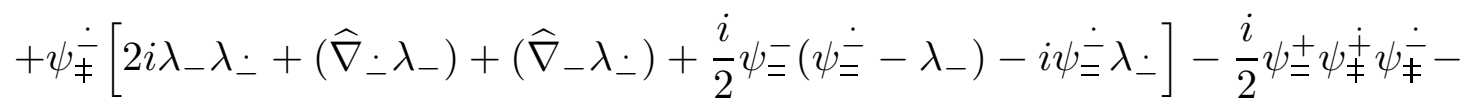

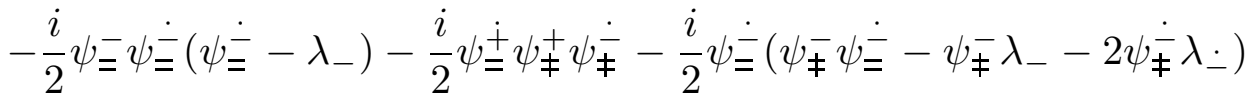




$$
\begin{aligned}
& \lambda_{\dot{+}} S-\lambda_{+} \bar{S}+i\left(\widehat{\nabla}_{\dot{+}} S\right)+i\left(\widehat{\nabla}_{+} \bar{S}\right)= \\
& =\left[\mathbf{D}_{\ddagger}, \mathbf{D}_{=}\right]_{\mathcal{X}}+\psi_{\mp}^{+}\left[\frac{i}{2}\left(\widehat{\nabla}_{-} R\right)+\frac{i}{2}\left(\widehat{\nabla}_{\dot{-}} F\right)-\frac{1}{2} \lambda_{-} H+\frac{1}{2} \lambda_{-} G-\frac{1}{4} \psi_{=}^{-} G-\frac{1}{4} \psi_{=}^{\dot{\dot{ }}} H\right]-\frac{1}{4} \psi_{\mp}^{-}\left(\psi_{=}^{+} \bar{H}+\right. \\
& \left.+\psi_{=}^{\dot{ \pm}} G\right)+\psi_{\mp}^{\dot{+}}\left[\frac{i}{2}\left(\widehat{\nabla}_{-} R\right)+\frac{i}{2}\left(\widehat{\nabla}_{\dot{-}} F\right)-\frac{1}{2} \lambda_{-} H+\frac{1}{2} \lambda_{-} G-\frac{1}{4} \psi_{=}^{-} G-\frac{1}{4} \psi_{=}^{\dot{-}} H\right]-\frac{1}{4} \psi_{\ddagger}^{\dot{-}}\left(\psi_{=}^{+} \bar{G}+\psi_{=}^{\dot{+}} H\right)+ \\
& +\frac{1}{4} \psi_{=}^{+}\left(\psi_{\ddagger}^{-} \bar{H}+\psi_{\ddagger}^{\dot{-}} \bar{G}\right)+\psi_{=}^{-}\left[\frac{i}{2}\left(\widehat{\nabla}_{\dot{+}} \bar{R}\right)+\frac{i}{2}\left(\widehat{\nabla}_{+} F\right)+\frac{1}{2}\left(\lambda_{\dot{+}}+\frac{1}{2} \psi_{\ddagger}^{+}\right) H-\frac{1}{2}\left(\lambda_{+}-\frac{1}{2} \psi_{\ddagger}^{\dot{+}}\right) G\right]+ \\
& +\frac{1}{4} \psi_{=}^{\dot{+}}\left(\psi_{\ddagger}^{-} G+\psi_{\ddagger}^{\dot{-}} H\right)-\psi_{=}^{\dot{\dot{\Xi}}}\left[\frac{i}{2}\left(\widehat{\nabla}_{+} R\right)+\frac{i}{2}\left(\widehat{\nabla}_{\dot{+}} \bar{F}\right)-\frac{1}{2} \lambda_{+} H+\frac{1}{2} \lambda_{\dot{+}} \bar{G}-\frac{1}{4} \psi_{\ddagger}^{+} \bar{G}-\frac{1}{4} \psi_{\ddagger}^{\dot{+}} \bar{H}\right]
\end{aligned}
$$

\section{Appendix D. Derivation of the projection formula in $\mathcal{N}=(2,2)$ dilaton super- gravity}

Here we repeat the derivation [6] of the local density projection formula in $\mathcal{N}=(2,2)$ dilaton supergravity. Namely, we start by writing the most general expression for the chiral projector with the right dimension and index structure:

$$
\int d^{2} x d^{4} \theta E^{-1} \mathcal{L}=\int d^{2} x e^{-1}\left[\widehat{\nabla}_{+} \widehat{\nabla}_{-}+X^{+} \widehat{\nabla}_{+}+X^{-} \widehat{\nabla}_{-}+Y\right] \widehat{\bar{\nabla}}^{2} \mathcal{L} \mid
$$

where the coefficients $X^{\alpha}$ and $Y$ are to be determined. Following [6], we evaluate (D.1) for the kinetic action $\mathcal{L}=\bar{\Phi} \Phi$ of a free chiral multiplet:

$$
\begin{aligned}
& \int d^{2} x d^{4} \theta E^{-1} \bar{\Phi} \Phi=\int d^{2} x e^{-1}\left[\left(\widehat{\nabla}^{2} \widehat{\bar{\nabla}}^{2} \bar{\Phi}\right) \Phi\left|+\left(\widehat{\nabla}_{+} \widehat{\bar{\nabla}}^{2} \bar{\Phi}\right)\left(\widehat{\nabla}_{-} \Phi\right)\right|-\left(\widehat{\nabla}_{-} \widehat{\bar{\nabla}}^{2} \bar{\Phi}\right)\left(\widehat{\nabla}_{+} \Phi\right) \mid+\right. \\
& +\left(\widehat{\bar{\nabla}}^{2} \bar{\Phi}\right)\left(\widehat{\nabla}^{2} \Phi\right)\left|+X^{+}\left(\widehat{\nabla}_{+} \widehat{\bar{\nabla}}^{2} \bar{\Phi}\right) \Phi\right|+X^{+}\left(\widehat{\bar{\nabla}}^{2} \bar{\Phi}\right)\left(\widehat{\nabla}_{+} \Phi\right) \mid \\
& \left.+X^{-}\left(\widehat{\nabla}_{-} \widehat{\bar{\nabla}}^{2} \bar{\Phi}\right) \Phi\left|+X^{-}\left(\widehat{\bar{\nabla}}^{2} \bar{\Phi}\right)\left(\widehat{\nabla}_{-} \Phi\right)\right|+Y\left(\widehat{\bar{\nabla}}^{2} \bar{\Phi}\right) \Phi \mid\right]
\end{aligned}
$$

Clearly, the left-hand side of this formula is invariant under complex conjugation. So, the

right-hand side must be invariant as well. As we will see in a moment, this condition completely determines the unknown coefficients $X^{\alpha}$ and $Y$. It suffices to consider only bosonic terms. Using the formulas in appendix $\mathrm{C}$ along with the definition (4.4), one can easily compute the relevant components:

$$
\begin{gathered}
\widehat{\nabla}_{+} \widehat{\nabla}_{\dot{+}} \widehat{\nabla}_{\dot{-}} \bar{\Phi} \mid \sim-\psi_{\mp}^{-} \mathbf{D}_{=} \bar{\phi}+\left(\psi_{\ddagger}^{\dot{+}}-\lambda_{+}\right) \bar{A} \\
\widehat{\nabla}_{-} \widehat{\nabla}_{\dot{+}} \widehat{\nabla}_{\dot{-}} \bar{\Phi} \mid \sim \psi_{=}^{+} \mathbf{D}_{\ddagger} \bar{\phi}+\left(\psi_{=}^{\dot{\Xi}}-\lambda_{-}\right) \bar{A}
\end{gathered}
$$




$$
\begin{aligned}
& \widehat{\nabla}_{+} \widehat{\nabla}_{-} \widehat{\nabla}_{\dot{+}} \widehat{\nabla}_{-} \bar{\Phi} \mid \sim \mathbf{D}_{=} \mathbf{D}_{\ddagger} \bar{\phi}+i\left(\psi_{=}^{\dot{ \pm}} \psi_{\ddagger}^{+}-\lambda_{+} \psi_{=}^{+}\right) \mathbf{D}_{\ddagger} \bar{\phi}+
\end{aligned}
$$

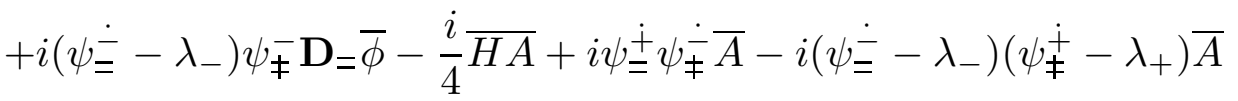

Substituting these into (D.2), we find that the resulting component action is invariant under complex conjugation only if we put:

$$
\begin{gathered}
X^{+}=i\left(\psi_{\stackrel{\dot{亠}}{=}}-\lambda_{-}\right) \\
X^{-}=-i\left(\psi_{\neq}^{\dot{+}}-\lambda_{+}\right) \\
Y=-\frac{1}{4} \bar{H}-\psi_{\ddagger}^{\dot{\dot{亠}}} \psi_{=}^{\dot{+}}+\left(\psi_{=}^{\dot{\doteq}}-\lambda_{-}\right)\left(\psi_{\ddagger}^{\dot{+}}-\lambda_{+}\right)
\end{gathered}
$$

With these expressions for $X^{\alpha}$ and $Y$ the result does not depend on whether we use the chiral projector (D.1) or its complex conjugate, of course, as it should be. Specifically, the chiral projection formula in $\mathcal{N}=(2,2)$ dilaton supergravity takes the following form:

$$
\begin{aligned}
\int d^{2} x d^{4} \theta E^{-1} \mathcal{L} & =\int d^{2} x e^{-1}\left[\widehat{\nabla}_{+} \widehat{\nabla}_{-}+i\left(\psi_{=}^{\dot{\doteq}}-\lambda_{-}\right) \widehat{\nabla}_{+}-i\left(\psi_{\mp}^{\dot{+}}-\lambda_{+}\right) \widehat{\nabla}_{-}+\right. \\
+ & \left.\left(-\frac{1}{4} \bar{H}-\psi_{\ddagger}^{\dot{\dot{亠}}} \psi_{=}^{\dot{ \pm}}+\left(\psi_{\doteq}^{\dot{\doteq}}-\lambda_{-}\right)\left(\psi_{\mp}^{\dot{+}}-\lambda_{+}\right)\right)\right] \widehat{\nabla}^{2} \mathcal{L} \mid
\end{aligned}
$$

One can easily check that this expression is equivalent to the chiral density projector (5.6) obtained by de-gauging the corresponding projector in the non-minimal $\mathcal{N}=(2,2)$ supergravity. 


\section{References}

[1] S.-T. Yau, editor, Essays on Mirror Manifolds, International Press, 1992; B. Greene and S.-T. Yau, editors, Mirror Symmetry. II, International Press, 1997.

[2] C. Vafa, "Evidence for F-Theory", Nucl. Phys. B496 (1996) 403.

[3] A. Klemm, "On the Geometry behind $\mathrm{N}=2$ Supersymmetric Effective Actions in Four Dimensions", hep-th/9705131.

[4] S. J. Gates, Jr., "Vector Multiplets and the Phases of $\mathrm{N}=2$ Theories in 2D Through the Looking Glass", Phys. Lett. B352 (1995) 43.

[5] B. de Wit, M.T. Grisaru, E. Rabinovici, H. Nicolai, "Two-loop finiteness of D=2 supergravity", Phys.Lett. B286 (1992) 78.

[6] M.T. Grisaru, M.E. Wehlau, "Superspace Measures, Invariant Actions, and Component Projection Formulae for (2,2) Supergravity", Nucl. Phys. B457 (1995) 219.

[7] A. Klemm, B. Lian, S.-S. Roan, S.-T. Yau, "Calabi-Yau fourfolds for M- and F-Theory compactifications", Nucl.Phys. B518 (1998) 515.

[8] P. Candelas, X. C. de la Ossa, "Moduli Space of Calabi-Yau Manifolds", Nucl.Phys. B355 (1991) 455.

[9] M. Haack, J. Louis, "Duality in Heterotic Vacua With Four Supercharges", hepth/9912181.

[10] S. Sethi, C. Vafa, and E. Witten, "Constraints on Low-Dimensional String Compactifications", Nucl. Phys. B480 (1996) 213.

[11] E. Witten, "On Flux Quantization In M-Theory And The Effective Action", J. Geom. Phys. 22 (1997) 1.

[12] A. Sevrin and J. Troost, "Off-Shell Formulation of N=2 Non-Linear Sigma-Models", Nucl.Phys. B492 (1997) 623.

[13] S. Gukov, C. Vafa and E. Witten, "CFT's From Calabi-Yau Four-folds", hepth/9906070.

[14] S. Gukov, "Solitons, Superpotentials and Calibrations", hep-th/9911011.

[15] W. Lerche, "Fayet-Iliopoulos Potentials from Four-Folds", JHEP 9711 (1997) 004.

[16] S.J. Gates, M.T. Grisaru, M. Rocek and W. Siegel, "Superspace", BenjaminCummings, 1983.

[17] P.S. Howe and G. Papadopoulos, "N=2, D = 2 Supergeometry", Class. Quantum Grav. 4 (1987) 11.

[18] S.J. Gates, C.M. Hull and M. Rocek, "Twisted Multiplets and New Supersymmetric Nonlinear Sigma Models", Nucl.Phys. B248 (1984) 157.

[19] K. Becker, M. Becker, "M-Theory on Eight-Manifolds", Nucl.Phys. B477 (1996) 155.

[20] W. Lerche, C. Vafa and N.P. Warner, "Chiral Rings in N=2 Superconformal Theories", Nucl.Phys. B324 (1989) 427. 
[21] L. Dixon, Lectures at the 1987 ICTP summer Workshop in High Energy Physics and Cosmology.

[22] S.J. Gates, Jr., M.T. Grisaru and M.E. Wehlau, "A Study of General 2D, N=2 Matter Coupled to Supergravity in Superspace", Nucl.Phys. B460 (1996) 579.

[23] S.J. Gates, T. Kadoyoshi, S. Nojiri, S.D. Odintsov, "Quantum cosmology in the models of 2d and 4d dilatonic supergravity with WZ matter", Phys.Rev. D58 (1998) 084026 .

[24] C. Callan, S. Giddings, J. Harvey, A. Strominger, "Evanescent Black Holes", Phys.Rev. D45 (1992) 1005.

[25] A. Alnowaiser, "Supergravity with $\mathrm{N}=2$ in Two Dimensions", Class. Quantum Grav. 7 (1990) 1033.

[26] M.T. Grisaru and M.E. Wehlau, "Prepotentials for $(2,2)$ Supergravity", Int. J. Mod. Phys. A10 (1995) 753.

[27] S.J. Gates, "Ectoplasm Has No Topology", Nucl.Phys. B541 (1999) 615.

[28] E. Bergshoeff, E. Sezgin, "The $(4,0)$ Heterotic String with Wess-Zumino Term", Mod. Phys. Lett. A1 (1986) 191.

[29] H. Nishino, "Alternative $N=(4,0)$ Superstring and $\sigma$-Models", Phys.Lett. B355 (1995) 117.

[30] S. J. Gates, Jr., L. Rana, "Manifest $(4,0)$ Supersymmetry, Sigma Models and The ADHM Instaton Construction", Phys. Lett. B345 (1995) 233.

[31] K. Dasgupta, S. Mukhi, "A Note on Low-Dimensional String Compactifications", Phys. Lett. B398 (1997) 285.

[32] C. M. Hull, G. Papadopoulos, P. K. Townsend, "Potentials for $(p, 0)$ and $(1,1)$ Supersymmetric Sigma Models with Torsion", Phys.Lett. B316 (1993) 291.

[33] R. Dhanawittayapol, S. J. Gates Jr., L. Rana, "A Canticle on $(4,0)$ Supergravity-Scalar Multiplet Systems for a Cognoscente", Phys.Lett. B389 (1996) 264. 